\title{
Vibrational analysis of boldine hydrochloride using QM/MM approach
}

\author{
Neeraj Misra ${ }^{\mathrm{a}, *}$, Shamoon Ahmad Siddiqui ${ }^{\mathrm{b}}$, Ruby Srivastava ${ }^{\mathrm{a}}$, Anoop Kumar Pandey ${ }^{\mathrm{a}}$ and \\ Sudha Jain $^{\mathrm{c}}$ \\ ${ }^{a}$ Department of Physics, Lucknow University, Lucknow, India \\ ${ }^{\mathrm{b}}$ Molecular Biophysics Unit, Indian institute of Science, Bangalore, India \\ ${ }^{\mathrm{c}}$ Department of Chemistry, Lucknow University, Lucknow, India
}

\begin{abstract}
A complete vibrational analysis was performed on the molecular structure of boldine hydrochloride using QM/MM method. The equilibrium geometry, harmonic vibrational frequencies and infrared intensities were calculated by QM/MM method with B3LYP/6-31G(d) and universal force field (UFF) combination using ONIOM code. We found the geometry obtained by the QM/MM method to be very accurate, and we can use this rapid method in place of time consuming ab initio methods for large molecules. A detailed interpretation of the infrared spectra of boldine hydrochloride is reported. The scaled theoretical wave numbers are in perfect agreement with the experimental values. The FT-IR spectra of boldine hydrochloride in the region 4000-500 $\mathrm{cm}^{-1}$ were recorded in CsI (solid phase) and in chloroform with concentration 5 and $10 \mathrm{mg} / \mathrm{ml}$.
\end{abstract}

Keywords: QM/MM method, FT-IR spectra, boldine hydrochloride, vibrational spectra

\section{Introduction}

Boldine hydrochloride (5,6,6a,7-tetrahydro-1,10-dimethoxy-6-methyl 4H-dibenzo quinoline-2,9-diol) is an aporphine alkaloid noted for its antioxidant properties [1]. Peumus boldus Mol. (Monimiaceae) also known as Boldo is an endemic Chilean tree known for its medicinal properties [16]. In addition to boldine, isolated more than 100 years ago, several aporphines and one benzylisoquinoline have been identified in the leaves $[10,20,26]$. Our title molecule is a aporphine alkaloid which has attracted our attention due to its potent antioxidant properties as it affords a highly stabilized free radical after reacting with species $\left(\mathrm{HO}_{\mathrm{Z}}\right)$, the degree of conjugation between the two aromatic rings, associated with the planarity of the biphenyl moiety, is a key factor in its antioxidant activity [1].

Here in the present communication, the experimental FT-IR frequencies of the title molecule are compared with the theoretical frequencies obtained by QM/MM method with B3LYP/6-31G(d) and universal force field (UFF) combination using ONIOM code. To gain a better understanding of the performance and limitation of QM/MM method, as a general approach to the vibrational problems of organic molecules, we calculated harmonic frequencies of boldine hydrochloride by this method and compared these results with the observed fundamental vibrational frequencies. Thus the evaluation of the advantages of this method is useful for obtaining a reliable assignment of the vibrational spectra. The purpose of this

\footnotetext{
${ }^{*}$ Corresponding author: Neeraj Misra, B-981, sector-A, Mahanagar, Lucknow 226006, India. Tel.: +91 0522 2335914; E-mail: neerajmisralu@ rediffmail.com.
} 
study is important because of further work on boldine hydrochloride, in which presumably some modification by chemical reaction such as oxidation/reduction on polyfunctional bioactive natural product should possibly generate a new reactive site in the molecule. The aim of this study is to check the performance of QM/MM method for simulation of infrared spectra of title compound with the use of standard B3LYP/6-31G(d) method and universal force field (UFF) combination. To the best of our knowledge neither the QM nor the MM calculations for boldine hydrochloride have been reported so far in the literature.

\section{Experimental section}

The FT-IR spectra of boldine hydrochloride has been recorded on a Perkin Elmer 1800 Spectrophotometer. Spectroscopic preparations of the sample were carried out under an atmosphere of prepurified nitrogen. The spectra were recorded for $1 \%$ and $5 \%$ solution of boldine. The FT-IR spectra of title molecule in solid phase (CsI) is shown in Fig. 1a and the FT-IR spectra of boldine hydrochloride in liquid phase (chloroform) using different concentration $(5$ and $10 \mathrm{mg} / \mathrm{ml})$ were also recorded and are shown in Fig. $1 b$ and $c$.

\section{Theoretical approach and computational details}

ONIOM is the hybrid method of Morokuma and co-workers that enables different levels of theory to be applied to different parts of a molecule/system and combined to produce a consistent energy expression.

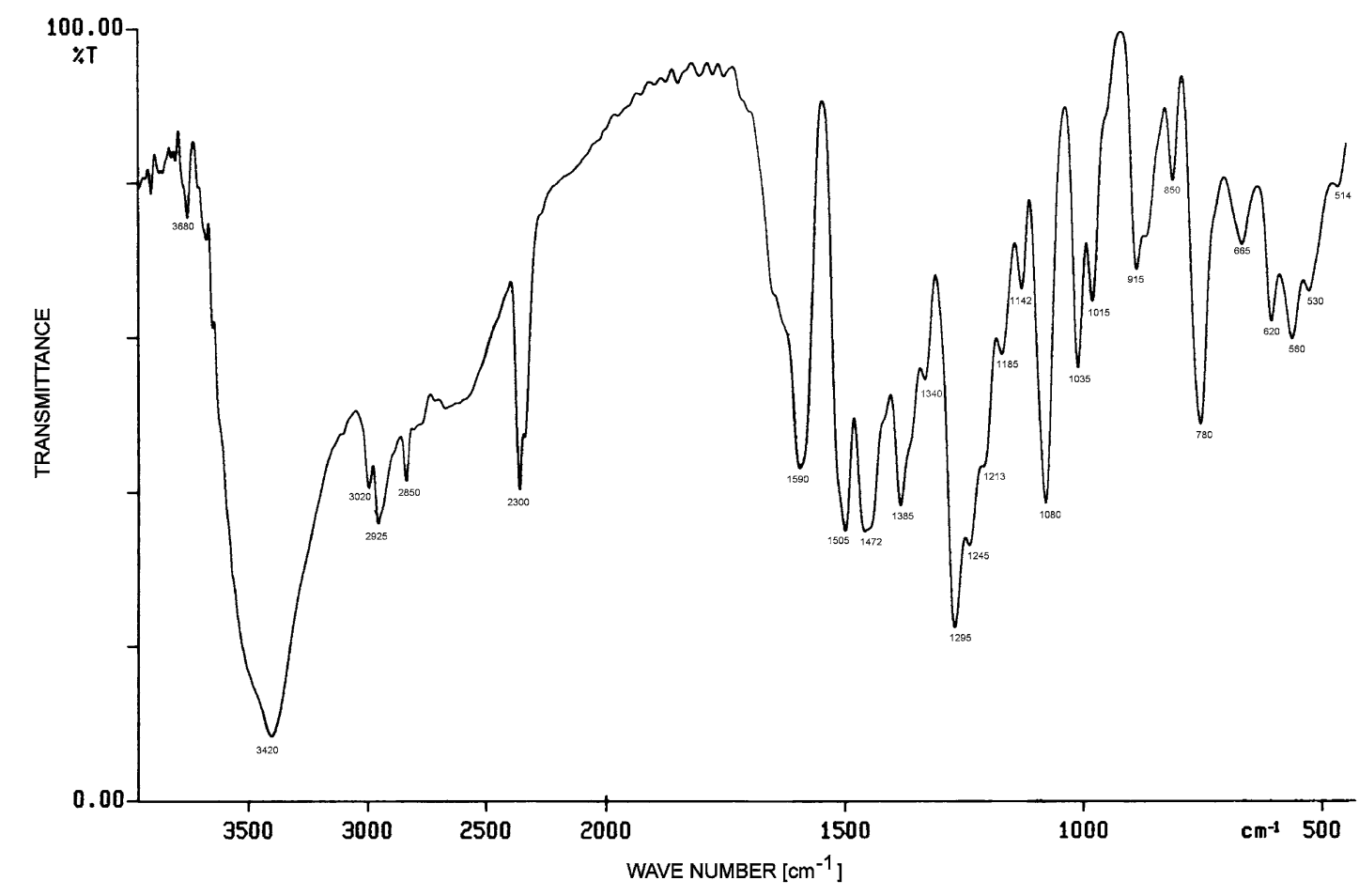

Fig. 1a. FT-IR spectra of boldine hydrochloride in CsI (solid phase). 


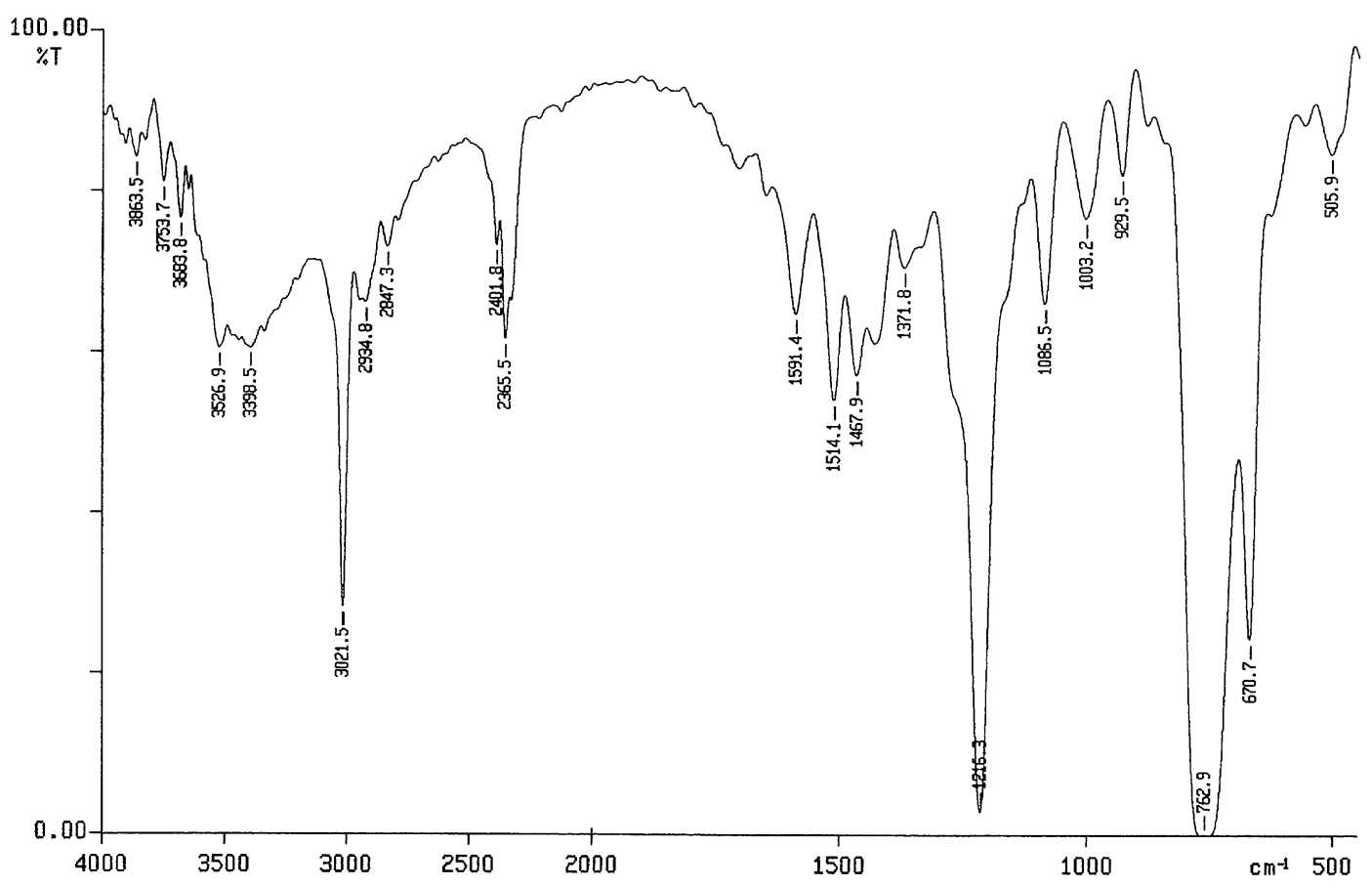

Fig. 1b. FT-IR spectra of boldine hydrochloride in chloroform $(5 \mathrm{mg} / \mathrm{ml})$.

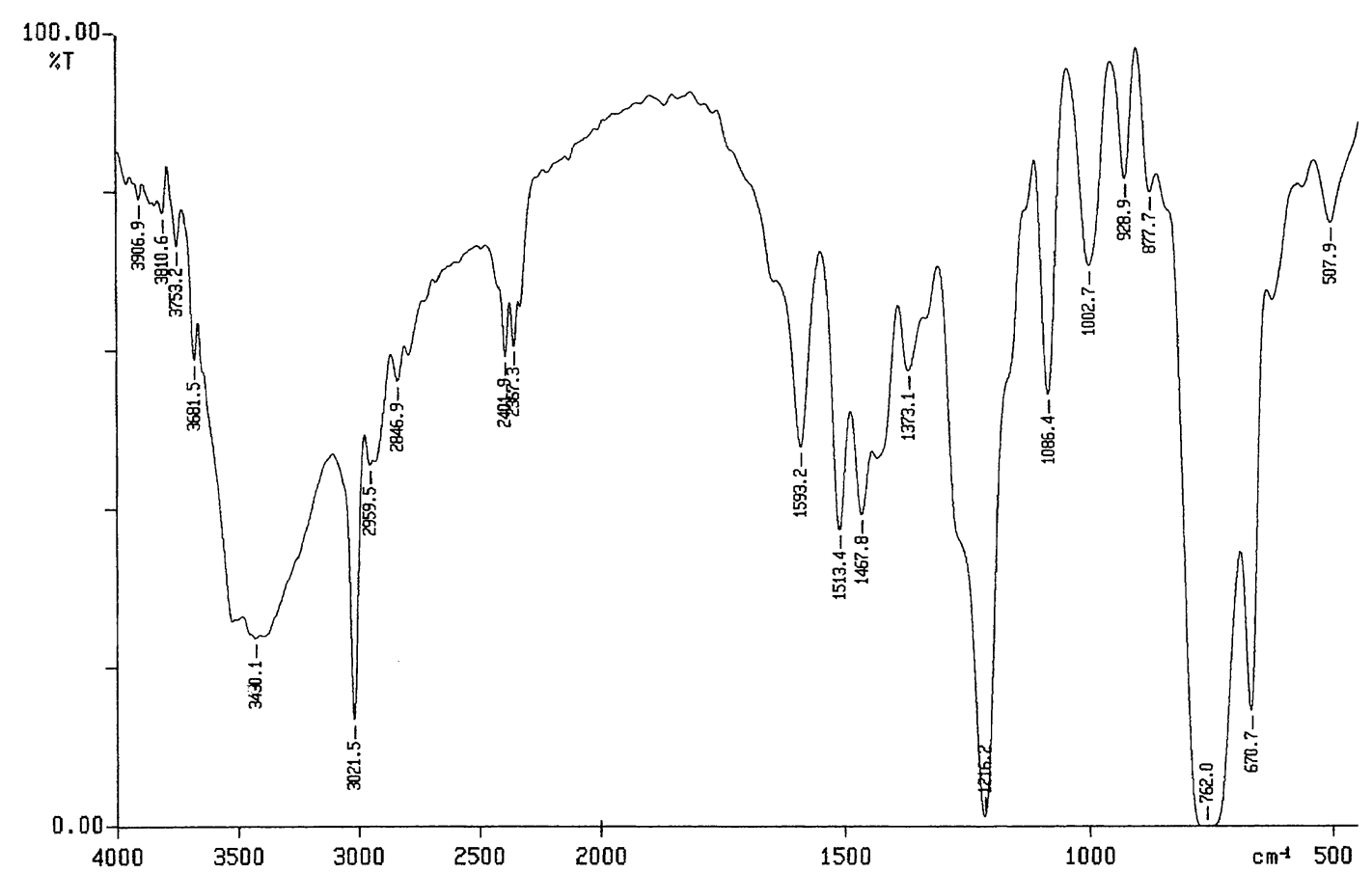

Fig. 1c. FT-IR spectra of boldine hydrochloride in chloroform $(10 \mathrm{mg} / \mathrm{ml})$. 


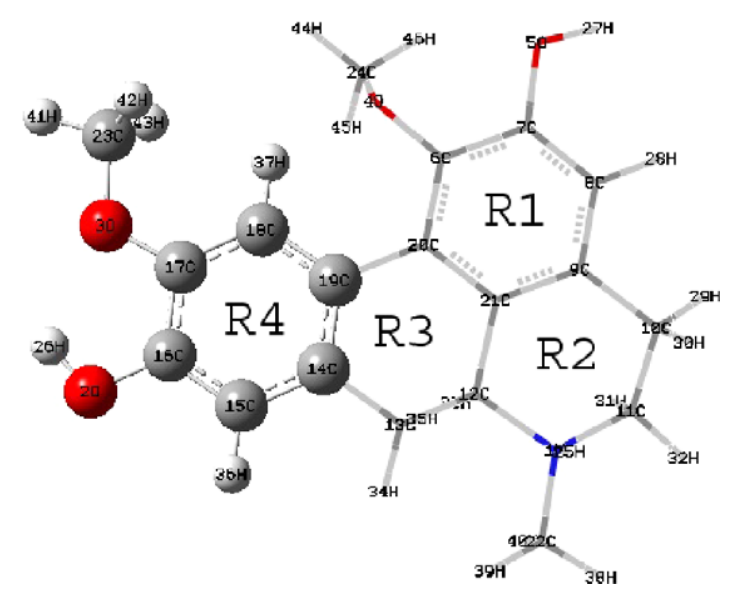

Fig. 2. Model molecular structure of boldine hydrochloride.

The objective is to perform a high-level calculation on just a small part of the system and to include the effects of the remainder at lower levels of theory, with the end result being of similar accuracy to a high-level calculation on the full system $[5,8,25]$.

Figure 2 shows the model molecular structure of boldine hydrochloride. In this molecule, atoms in ring R4 and its adjacent atoms are treated quantum mechanically and these atoms are shown by balls and sticks in Fig. 2, while the remaining part of the molecule is treated by molecular mechanics method. In molecular mechanics method we have used universal force field (UFF) [19]. UFF correctly predicts the structures of unstrained and uncongested hydrocarbons, silanes, alkenes, saturated amines, saturated ethers and phosphines, aromatic systems, and simple unconjugated multiple bond containing compounds such as nitriles, ketones, and imines well [4].

The entire calculations were performed on a Pentium IV/1.66 GHz personal computer using Gaussian 03W [7] program package, invoking gradient geometry optimization [21]. Initial geometry was generated from standard geometrical parameters and was minimized without any constraint in the potential energy surface at B3LYP level and UFF combination, adopting the standard 6-31G(d) basis set. The optimized structural parameters were used in the vibrational frequency calculations to characterize all the stationary points as minima. We have utilized the gradient corrected density functional theory (DFT) [9] with the three-parameter hybrid functional (B3LYP) [2] for the exchange part and the Lee-Yang-Parr (LYP) correlation function [17], for the computation of molecular structure, vibrational frequencies, and energies of the optimized structures. Density functional theory offers electron correlation which is frequently comparable to second-order Moller-Plesset theory (MP2) [22,23]. Vibrational frequencies calculated at B3LYP/6-31G(d) level and UFF combination below $800 \mathrm{~cm}^{-1}$ were scaled by a factor of 1.0013 and above $800 \mathrm{~cm}^{-1}$ were scaled by a factor of 0.9613 [24]. By combining the result of GAUSSVIEW program [6] with symmetry considerations, vibrational frequency assignments were made with a high degree of accuracy. There is always some ambiguity in defining internal coordination. However, the defined coordinate form complete set and matches quite well with the motions observed using the GAUSSVIEW program. 


\section{Result and discussion}

\subsection{Geometry optimization}

The optimized structural parameters of boldine hydrochloride calculated by QM/MM with B3LYP method and UFF combination with the 6-31G(d) basis set are listed in Table 1 in accordance with the atom numbering scheme given in Fig. 2. Experimental values of various bond lengths and bond angles of boldine hydrochloride were taken from the literature [1]. For example, the optimized bond lengths of $\mathrm{C}-\mathrm{C}$ in ring $\mathrm{R} 1$ fall in the range from 1.390 to $1.426 \AA$, in good agreement with those of the experimental bond lengths [1.375-1.422 $\AA$ ] . The optimized bond lengths of $\mathrm{C}-\mathrm{C}$ in ring $\mathrm{R} 2$ fall in the range from 1.396 to $1.518 \AA$, also in good agreement with those of the experimental bond lengths [1.386-1.513 $\AA$ ]. The optimized bond lengths of $\mathrm{C}-\mathrm{C}$ in ring $\mathrm{R} 3$ fall in the range from 1.407 to $1.530 \AA$, also in good agreement with those of the experimental bond lengths [1.393-1.520 ̊]. The optimized bond lengths of $\mathrm{C}-\mathrm{C}$ in ring $\mathrm{R} 4$ fall in the range from 1.387 to $1.413 \AA$, also in good agreement with those of the experimental bond lengths [1.375-1.416 $\AA$ ]. The optimized bond lengths of $\mathrm{C}-\mathrm{N}$ fall in the range from 1.502 to $1.534 \AA$, also in good agreement with those of the experimental bond lengths [1.493-1.508 $\AA$ ]. The optimized bond lengths of $\mathrm{C}-\mathrm{O}$ fall in the range from 1.351 to $1.439 \AA$, also in good agreement with those of the experimental bond lengths [1.359-1.428 $\mathrm{A}$. The values of other calculated bond lengths and bond angles are given in Table 1 and they are in excellent agreement with experimental values. Thus, although there are some difference between the theoretical values and experimental values, the optimized structural parameters can well reproduce the experimental ones and they are the basis for the thereafter discussion.

\subsection{Vibrational assignments}

Boldine hydrochloride has 46 atoms and 132 normal modes of fundamental vibration. All of the 132 fundamental vibrations are IR active. Detailed description of vibrational modes can be given by means of normal coordinate analysis. The detailed vibrational assignments can be analysed by comparing the band positions and intensities observed in FT-IR spectra with the wave numbers and intensities obtained from the QM/MM calculations given in Table 2 .

The experimental FT-IR spectra are shown in Fig. 1a-c. The descriptions concerning the assignment have been listed in Table 2. Gauss View Program [6] was used for the assignment of the calculated frequencies. The observed frequencies for various modes of vibrations are also presented in Table 2 .

\section{3. $C-H$ vibrations}

The hetero aromatic structure shows the presence of $\mathrm{C}-\mathrm{H}$ stretching vibrations in the region 3000 $3100 \mathrm{~cm}^{-1}$, which is the characteristic region for the ready identification of the $\mathrm{C}-\mathrm{H}$ stretching vibration [14]. In the present study the $\mathrm{C}-\mathrm{H}$ stretching vibrations are calculated at 3157, 3083, 3053 and $2928 \mathrm{~cm}^{-1}$, which are supported by the literature [14]. Some bending vibrations of C-H are also calculated and supported by the literature [14].

\subsection{Methylene group vibrations}

The asymmetric $\mathrm{CH}_{2}$ stretching vibrations are generally observed in the region $3100-3000 \mathrm{~cm}^{-1}$, while the symmetric stretching vibrations are generally observed between $3000-2900 \mathrm{~cm}^{-1}$ [15]. The 
Table 1

Optimized geometrical parameters of boldine hydrochloride

\begin{tabular}{|c|c|c|c|c|c|c|}
\hline S. No. & Bond lengths $(\AA)$ & X-ray & Calculated & Bond angles (degree) & X-ray & Calculated \\
\hline 1 & N1-C11 & 1.493 & 1.515 & C11-N1-C12 & 110.6 & 110.7 \\
\hline 2 & $\mathrm{~N} 1-\mathrm{C} 12$ & 1.508 & 1.534 & $\mathrm{C} 11-\mathrm{N} 1-\mathrm{C} 22$ & 109.7 & 110.9 \\
\hline 3 & $\mathrm{~N} 1-\mathrm{C} 22$ & 1.496 & 1.502 & $\mathrm{C} 11-\mathrm{N} 1-\mathrm{H} 25$ & & 107.3 \\
\hline 4 & $\mathrm{~N} 1-\mathrm{H} 25$ & & 1.027 & $\mathrm{C} 12-\mathrm{N} 1-\mathrm{C} 22$ & 111.6 & 113.3 \\
\hline 5 & $\mathrm{O} 2-\mathrm{C} 16$ & 1.370 & 1.351 & $\mathrm{C} 12-\mathrm{N} 1-\mathrm{H} 25$ & & 106.5 \\
\hline 6 & $\mathrm{O} 2-\mathrm{H} 26$ & & 0.975 & $\mathrm{C} 22-\mathrm{N} 1-\mathrm{H} 25$ & & 107.9 \\
\hline 7 & $\mathrm{O} 3-\mathrm{C} 17$ & 1.379 & 1.365 & $\mathrm{C} 16-\mathrm{O} 2-\mathrm{H} 26$ & & 107.6 \\
\hline 8 & $\mathrm{O} 3-\mathrm{C} 23$ & 1.411 & 1.426 & $\mathrm{C} 17-\mathrm{O} 3-\mathrm{C} 23$ & 117.8 & 118.7 \\
\hline 9 & O4-C6 & 1.385 & 1.367 & C6-O4-C24 & 115.1 & 116.1 \\
\hline 10 & $\mathrm{O} 4-\mathrm{C} 24$ & 1.428 & 1.439 & $\mathrm{C} 7-\mathrm{O} 5-\mathrm{H} 27$ & & 109.8 \\
\hline 11 & O5-C7 & 1.359 & 1.357 & O4-C6-C7 & 118.2 & 118.4 \\
\hline 12 & $\mathrm{O} 5-\mathrm{H} 27$ & & 0.971 & $\mathrm{O} 4-\mathrm{C} 6-\mathrm{C} 20$ & 121.1 & 121.0 \\
\hline 13 & C6-C7 & 1.401 & 1.413 & C7-C6-C20 & 120.7 & 120.5 \\
\hline 14 & C6-C20 & 1.398 & 1.407 & O5-C7-C6 & 116.3 & 116.6 \\
\hline 15 & C7-C8 & 1.375 & 1.390 & $\mathrm{O} 5-\mathrm{C} 7-\mathrm{C} 8$ & 123.5 & 123.2 \\
\hline 16 & C8-C9 & 1.397 & 1.398 & C6-C7-C8 & 120.1 & 120.3 \\
\hline 17 & C8-H28 & & 1.088 & C7-C8-C9 & 120.4 & 120.3 \\
\hline 18 & C9-C10 & 1.507 & 1.515 & C7-C8-H28 & & 119.8 \\
\hline 19 & C9-C21 & 1.386 & 1.396 & C9-C8-H28 & & 119.9 \\
\hline 20 & $\mathrm{C} 10-\mathrm{C} 11$ & 1.513 & 1.518 & C8-C9-C10 & 118.8 & 118.7 \\
\hline 21 & $\mathrm{C} 10-\mathrm{H} 29$ & & 1.096 & C8-C9-C21 & 120.0 & 119.9 \\
\hline 22 & C10-H30 & & 1.100 & C10-C9-C21 & 121.2 & 121.5 \\
\hline 23 & C11-H31 & & 1.094 & C9-C10-C11 & 113.5 & 112.2 \\
\hline 24 & $\mathrm{C} 11-\mathrm{H} 32$ & & 1.092 & C9-C10-H29 & & 110.5 \\
\hline 25 & $\mathrm{C} 12-\mathrm{C} 13$ & 1.518 & 1.530 & C9-C10-H30 & & 110.8 \\
\hline 26 & $\mathrm{C} 12-\mathrm{C} 21$ & 1.520 & 1.521 & C11-C10-H29 & & 107.8 \\
\hline 27 & C12-H33 & & 1.099 & C11-C10-H30 & & 109.5 \\
\hline 28 & $\mathrm{C} 13-\mathrm{C} 14$ & 1.508 & 1.512 & H29-C10-H30 & & 105.7 \\
\hline 29 & C13-H34 & & 1.093 & N1-C11-C10 & 111.1 & 109.5 \\
\hline 30 & C13-H35 & & 1.102 & N1-C11-H31 & & 106.0 \\
\hline 31 & $\mathrm{C} 14-\mathrm{C} 15$ & 1.392 & 1.396 & N1-C11-H32 & & 107.6 \\
\hline 32 & C14-C19 & 1.393 & 1.407 & C10-C11-H31 & & 112.3 \\
\hline 33 & $\mathrm{C} 15-\mathrm{C} 16$ & 1.375 & 1.390 & C10-C11-H32 & & 112.2 \\
\hline 34 & C15-H36 & & 1.086 & H31-C11-H32 & & 108.9 \\
\hline 35 & $\mathrm{C} 16-\mathrm{C} 17$ & 1.396 & 1.413 & N1-C12-C13 & 110.0 & 111.2 \\
\hline 36 & $\mathrm{C} 17-\mathrm{C} 18$ & 1.383 & 1.387 & $\mathrm{~N} 1-\mathrm{C} 12-\mathrm{C} 21$ & 110.6 & 110.9 \\
\hline 37 & C18-C19 & 1.416 & 1.413 & $\mathrm{~N} 1-\mathrm{C} 12-\mathrm{H} 33$ & & 104.0 \\
\hline 38 & C18-H37 & & 1.079 & C13-C12-C21 & 109.6 & 109.9 \\
\hline 39 & C19-C20 & 1.486 & 1.484 & C13-C12-H33 & & 109.2 \\
\hline 40 & $\mathrm{C} 20-\mathrm{C} 21$ & 1.422 & 1.426 & C21-C12-H33 & & 111.5 \\
\hline 41 & $\mathrm{C} 22-\mathrm{H} 38$ & & 1.091 & C12-C13-C14 & 109.0 & 107.5 \\
\hline 42 & C22-H39 & & 1.088 & C12-C13-H34 & & 112.1 \\
\hline 43 & $\mathrm{C} 22-\mathrm{H} 40$ & & 1.091 & C12-C13-H35 & & 109.0 \\
\hline 44 & $\mathrm{C} 23-\mathrm{H} 41$ & & 1.090 & C14-C13-H34 & & 109.1 \\
\hline 45 & $\mathrm{C} 23-\mathrm{H} 42$ & & 1.096 & C14-C13-H35 & & 111.4 \\
\hline
\end{tabular}


Table 1

(Continued)

\begin{tabular}{|c|c|c|c|c|c|c|}
\hline S. No. & Bond lengths $(\AA)$ & X-ray & Calculated & Bond angles (degree) & $\mathrm{X}$-ray & Calculated \\
\hline 46 & $\mathrm{C} 23-\mathrm{H} 43$ & & 1.096 & H34-C13-H35 & & 107.8 \\
\hline 47 & $\mathrm{C} 24-\mathrm{H} 44$ & & 1.091 & C13-C14-C15 & 119.7 & 120.0 \\
\hline 48 & $\mathrm{C} 24-\mathrm{H} 45$ & & 1.096 & C13-C14-C19 & 119.9 & 119.1 \\
\hline 49 & $\mathrm{C} 24-\mathrm{H} 46$ & & 1.093 & C15-C14-C19 & 120.3 & 120.9 \\
\hline 50 & & & & $\mathrm{C} 14-\mathrm{C} 15-\mathrm{C} 16$ & 120.8 & 120.4 \\
\hline 51 & & & & $\mathrm{C} 14-\mathrm{C} 15-\mathrm{H} 36$ & & 121.2 \\
\hline 52 & & & & C16-C15-H36 & & 118.3 \\
\hline 53 & & & & $\mathrm{O} 2-\mathrm{C} 16-\mathrm{C} 15$ & 117.8 & 120.2 \\
\hline 54 & & & & $\mathrm{O} 2-\mathrm{C} 16-\mathrm{C} 17$ & 122.5 & 120.6 \\
\hline 55 & & & & $\mathrm{C} 15-\mathrm{C} 16-\mathrm{C} 17$ & 119.8 & 119.2 \\
\hline 56 & & & & $\mathrm{O} 3-\mathrm{C} 17-\mathrm{C} 16$ & 114.6 & 113.3 \\
\hline 57 & & & & O3-C17-C18 & 125.3 & 126.2 \\
\hline 58 & & & & C16-C17-C18 & 120.1 & 120.5 \\
\hline 59 & & & & C17-C18-C19 & 120.5 & 120.6 \\
\hline 60 & & & & C17-C18-H37 & & 120.2 \\
\hline 61 & & & & C19-C18-H37 & & 119.3 \\
\hline 62 & & & & C14-C19-C18 & 118.4 & 118.3 \\
\hline 63 & & & & C14-C19-C20 & 118.0 & 118.5 \\
\hline 64 & & & & C18-C19-C20 & 123.5 & 123.2 \\
\hline 65 & & & & C6-C20-C19 & 123.3 & 123.1 \\
\hline 66 & & & & C6-C20-C21 & 118.2 & 118.0 \\
\hline 67 & & & & C19-C20-C21 & 118.4 & 118.9 \\
\hline 68 & & & & C9-C21-C12 & 122.3 & 122.6 \\
\hline 69 & & & & C9-C21-C20 & 120.4 & 121.0 \\
\hline 70 & & & & $\mathrm{C} 12-\mathrm{C} 21-\mathrm{C} 20$ & 117.3 & 116.4 \\
\hline 71 & & & & N1-C22-H38 & & 108.5 \\
\hline 72 & & & & N1-C22-H39 & & 110.0 \\
\hline 73 & & & & N1-C22-H40 & & 108.4 \\
\hline 74 & & & & H38-C22-H39 & & 109.8 \\
\hline 75 & & & & $\mathrm{H} 38-\mathrm{C} 22-\mathrm{H} 40$ & & 110.0 \\
\hline 76 & & & & H39-C22-H40 & & 110.1 \\
\hline 77 & & & & O3-C23-H41 & & 105.9 \\
\hline 78 & & & & $\mathrm{O} 3-\mathrm{C} 23-\mathrm{H} 42$ & & 111.0 \\
\hline 79 & & & & O3-C23-H43 & & 111.0 \\
\hline 80 & & & & $\mathrm{H} 41-\mathrm{C} 23-\mathrm{H} 42$ & & 109.7 \\
\hline 81 & & & & $\mathrm{H} 41-\mathrm{C} 23-\mathrm{H} 43$ & & 109.6 \\
\hline 82 & & & & $\mathrm{H} 42-\mathrm{C} 23-\mathrm{H} 43$ & & 109.6 \\
\hline 83 & & & & O4-C24-H44 & & 105.7 \\
\hline 84 & & & & $\mathrm{O} 4-\mathrm{C} 24-\mathrm{H} 45$ & & 110.4 \\
\hline 85 & & & & O4-C24-H46 & & 111.1 \\
\hline 86 & & & & $\mathrm{H} 44-\mathrm{C} 24-\mathrm{H} 45$ & & 109.6 \\
\hline 87 & & & & $\mathrm{H} 44-\mathrm{C} 24-\mathrm{H} 46$ & & 109.5 \\
\hline 88 & & & & $\mathrm{H} 45-\mathrm{C} 24-\mathrm{H} 46$ & & 110.3 \\
\hline
\end{tabular}


Table 2

Vibrational wave numbers obtained for boldine hydrochloride in $\mathrm{cm}^{-1}$, experimental frequencies from FT-IR spectra in $\mathrm{cm}^{-1}$, IR intensities $\left(K_{m} \mathrm{~mol}^{-1}\right)$ and assignment

\begin{tabular}{|c|c|c|c|c|c|c|c|}
\hline \multirow[t]{2}{*}{ S. No. } & \multicolumn{2}{|c|}{ Wave number } & \multirow{2}{*}{$\begin{array}{l}\text { Exp. IR freq. } \\
\text { in solid phase }\end{array}$} & \multirow{2}{*}{$\begin{array}{l}\text { Exp. IR freq. } \\
(5 \mathrm{mg} / \mathrm{ml}) \text { in } \\
\mathrm{CHCl}_{3}\end{array}$} & \multirow{2}{*}{$\begin{array}{l}\text { Exp. IR freq. } \\
(10 \mathrm{mg} / \mathrm{ml}) \text { in } \\
\mathrm{CHCl}_{3}\end{array}$} & \multirow{2}{*}{$\begin{array}{c}\text { IR } \\
\text { intensity }\end{array}$} & \multirow[t]{2}{*}{ Assignment } \\
\hline & Unscal. & Scal. & & & & & \\
\hline 1 & 40 & 40 & & & & 2 & $\begin{array}{l}\tau(\mathrm{CC}) \mathrm{R} 1, \mathrm{R} 2, \mathrm{R} 3 \& \mathrm{R} 4+\tau(\mathrm{CN})+ \\
\tau(\mathrm{CO}) \mathrm{R} 4\end{array}$ \\
\hline 2 & 44 & 44 & & & & 3 & $\tau(\mathrm{CC}) \mathrm{R} 1, \mathrm{R} 2, \mathrm{R} 3 \& \mathrm{R} 4+\tau(\mathrm{CO}) \mathrm{R} 1 \& \mathrm{R} 4$ \\
\hline 3 & 59 & 59 & & & & 2 & $\tau(\mathrm{CO}) \mathrm{R} 1+\tau(\mathrm{CC}) \mathrm{R} 1, \mathrm{R} 2, \mathrm{R} 3 \& \mathrm{R} 4$ \\
\hline 4 & 80 & 80 & & & & 2 & $\begin{array}{l}\tau(\mathrm{CO}) \mathrm{R} 1+\gamma(\mathrm{COC}) \mathrm{R} 1+ \\
\tau(\mathrm{CN})+\tau(\mathrm{CC}) \mathrm{R} 1 \& \mathrm{R} 2\end{array}$ \\
\hline 5 & 83 & 83 & & & & 2 & $\begin{array}{l}\tau(\mathrm{CO}) \mathrm{R} 1 \& \mathrm{R} 4+\gamma(\mathrm{COC}) \mathrm{R} 4 \& \mathrm{R} 1+ \\
\tau(\mathrm{CC}) \mathrm{R} 2, \mathrm{R} 3 \& \mathrm{R} 4\end{array}$ \\
\hline 6 & 98 & 98 & & & & 3 & $\begin{array}{l}\tau(\mathrm{CO}) \mathrm{R} 1 \& \mathrm{R} 4+\gamma(\mathrm{COC}) \mathrm{R} 4 \& \mathrm{R} 1+ \\
\tau(\mathrm{CC}) \mathrm{R} 3 \& \mathrm{R} 4\end{array}$ \\
\hline 7 & 117 & 117 & & & & 2 & $\tau(\mathrm{CC}) \mathrm{R} 1, \mathrm{R} 2, \mathrm{R} 3 \& \mathrm{R} 4$ \\
\hline 8 & 142 & 142 & & & & 0 & $\tau(\mathrm{CO}) \mathrm{R} 1 \& \mathrm{R} 4+\tau(\mathrm{CC}) \mathrm{R} 3 \& \mathrm{R} 4$ \\
\hline 9 & 164 & 164 & & & & 1 & $\begin{array}{l}\tau(\mathrm{CN})+\tau(\mathrm{CO}) \mathrm{R} 4 \& \mathrm{R} 1+ \\
\tau(\mathrm{CC}) \mathrm{R} 1, \mathrm{R} 2, \mathrm{R} 3 \& \mathrm{R} 4+\gamma(\mathrm{CCC}) \mathrm{R} 3\end{array}$ \\
\hline 10 & 177 & 177 & & & & 0 & $\begin{array}{l}\tau(\mathrm{CO}) \mathrm{R} 4 \& \mathrm{R} 1+\tau(\mathrm{CN})+ \\
\tau(\mathrm{CC}) \mathrm{R} 3 \& \mathrm{R} 4\end{array}$ \\
\hline 11 & 182 & 182 & & & & 1 & $\begin{array}{l}\tau(\mathrm{CN})+\gamma(\mathrm{CCN})+\tau(\mathrm{CC}) \mathrm{R} 1 \& \mathrm{R} 2+ \\
\tau(\mathrm{CO}) \mathrm{R} 4 \& \mathrm{R} 1+\gamma(\mathrm{CNC})\end{array}$ \\
\hline 12 & 200 & 200 & & & & 2 & $\begin{array}{l}\tau(\mathrm{CO}) \mathrm{R} 4 \& \mathrm{R} 1+ \\
\tau(\mathrm{CC}) \mathrm{R} 1, \mathrm{R} 2, \mathrm{R} 3 \& \mathrm{R} 4+ \\
\gamma(\mathrm{COC}) \mathrm{R} 1 \& \mathrm{R} 4+\tau(\mathrm{CN})\end{array}$ \\
\hline 13 & 220 & 220 & & & & 2 & $\begin{array}{l}\tau(\mathrm{CN})+\tau(\mathrm{CC}) \mathrm{R} 2, \mathrm{R} 3 \& \mathrm{R} 4+ \\
\tau(\mathrm{CO}) \mathrm{R} 4 \& \mathrm{R} 1\end{array}$ \\
\hline 14 & 233 & 233 & & & & 1 & $\begin{array}{l}\tau(\mathrm{CO}) \mathrm{R} 4+\tau(\mathrm{CC}) \mathrm{R} 2, \mathrm{R} 3 \& \mathrm{R} 4+ \\
\gamma(\mathrm{CCC}) \mathrm{R} 2 \& \mathrm{R} 3\end{array}$ \\
\hline 15 & 235 & 235 & & & & 3 & $\begin{array}{l}\tau(\mathrm{CO}) \mathrm{R} 4 \& \mathrm{R} 1+\tau(\mathrm{CC}) \mathrm{R} 1, \mathrm{R} 2 \& \mathrm{R} 3+ \\
\gamma(\mathrm{CCC}) \mathrm{R} 1+\gamma(\mathrm{COC}) \mathrm{R} 1\end{array}$ \\
\hline 16 & 245 & 245 & & & & 0 & $\tau(\mathrm{CC}) \mathrm{R} 1 \& \mathrm{R} 2+\gamma(\mathrm{CCC}) \mathrm{R} 1 \& \mathrm{R} 2$ \\
\hline 17 & 275 & 275 & & & & 1 & $\begin{array}{l}\tau(\mathrm{CN})+\tau(\mathrm{CO}) \mathrm{R} 4 \& \mathrm{R} 1+ \\
\tau(\mathrm{CC}) \mathrm{R} 1, \mathrm{R} 2 \& \mathrm{R} 3\end{array}$ \\
\hline 18 & 293 & 293 & & & & 1 & $\begin{array}{l}\tau(\mathrm{CO}) \mathrm{R} 4+\gamma(\mathrm{CCC}) \mathrm{R} 1, \mathrm{R} 3 \& \mathrm{R} 4+ \\
\tau(\mathrm{CC}) \mathrm{R} 1, \mathrm{R} 3 \& \mathrm{R} 4\end{array}$ \\
\hline 19 & 303 & 303 & & & & 5 & $\tau(\mathrm{CO}) \mathrm{R} 4+\gamma(\mathrm{COC}) \mathrm{R} 3+\tau(\mathrm{CC}) \mathrm{R} 4$ \\
\hline 20 & 312 & 312 & & & & 10 & $\tau(\mathrm{CC}) \mathrm{R} 1, \mathrm{R} 2, \mathrm{R} 3 \& \mathrm{R} 4+\tau(\mathrm{CN})$ \\
\hline 21 & 337 & 337 & & & & 1 & $\begin{array}{l}\tau(\mathrm{CO}) \mathrm{R} 1+\gamma(\mathrm{COC}) \mathrm{R} 1+ \\
\beta(\mathrm{CCO}) \mathrm{R} 1+\tau(\mathrm{CC}) \mathrm{R} 1, \mathrm{R} 2, \mathrm{R} 3 \& \mathrm{R} 4\end{array}$ \\
\hline 22 & 356 & 356 & & & & 10 & $\begin{array}{l}\gamma(\mathrm{COC}) \mathrm{R} 1 \& \mathrm{R} 4+\tau(\mathrm{CO}) \mathrm{R} 4 \& \mathrm{R} 1+ \\
\tau(\mathrm{CC}) \mathrm{R} 1, \mathrm{R} 2, \mathrm{R} 3 \& \mathrm{R} 4+\gamma(\mathrm{CNC})+ \\
\gamma(\mathrm{CCN})\end{array}$ \\
\hline 23 & 377 & 377 & & & & 53 & $\begin{array}{l}\gamma(\mathrm{OH}) \mathrm{R} 1+\tau(\mathrm{CC}) \mathrm{R} 1, \mathrm{R} 2, \mathrm{R} 3 \& \mathrm{R} 4+ \\
\tau(\mathrm{CO}) \mathrm{R} 1\end{array}$ \\
\hline 24 & 383 & 383 & & & & 8 & $\begin{array}{l}\gamma(\mathrm{OH}) \mathrm{R} 1+\tau(\mathrm{CO}) \mathrm{R} 4+\tau(\mathrm{CN})+ \\
\gamma(\mathrm{COC}) \mathrm{R} 4+\tau(\mathrm{CC}) \mathrm{R} 2, \mathrm{R} 3 \& \mathrm{R} 4\end{array}$ \\
\hline
\end{tabular}


Table 2

(Continued)

\begin{tabular}{|c|c|c|c|c|c|c|c|}
\hline \multirow[t]{2}{*}{ S. No. } & \multicolumn{2}{|c|}{ Wave number } & \multirow{2}{*}{$\begin{array}{l}\text { Exp. IR freq. } \\
\text { in solid phase }\end{array}$} & \multirow{2}{*}{$\begin{array}{l}\text { Exp. IR freq. } \\
(5 \mathrm{mg} / \mathrm{ml}) \text { in } \\
\mathrm{CHCl}_{3}\end{array}$} & \multirow{2}{*}{$\begin{array}{l}\text { Exp. IR freq. } \\
(10 \mathrm{mg} / \mathrm{ml}) \text { in } \\
\mathrm{CHCl}_{3}\end{array}$} & \multirow{2}{*}{$\begin{array}{c}\text { IR } \\
\text { intensity }\end{array}$} & \multirow[t]{2}{*}{ Assignment } \\
\hline & Unscal. & Scal. & & & & & \\
\hline 25 & 390 & 390 & & & & 45 & $\begin{array}{l}\gamma(\mathrm{OH}) \mathrm{R} 1+\tau(\mathrm{CN})+ \\
\tau(\mathrm{CC}) \mathrm{R} 2, \mathrm{R} 3 \& \mathrm{R} 4\end{array}$ \\
\hline 26 & 400 & 400 & & & & 4 & $\begin{array}{l}\tau(\mathrm{CO}) \mathrm{R} 4 \& \mathrm{R} 1+ \\
\tau(\mathrm{CC}) \mathrm{R} 1, \mathrm{R} 2, \mathrm{R} 3 \& \mathrm{R} 4+ \\
\gamma(\mathrm{CCC}) \mathrm{R} 1, \mathrm{R} 2 \& \mathrm{R} 3+ \\
\tau(\mathrm{CN})+\gamma(\mathrm{CNC})+\gamma(\mathrm{CCN})\end{array}$ \\
\hline 27 & 437 & 437 & & & & 4 & $\begin{array}{l}\tau(\mathrm{CC}) \mathrm{R} 4, \mathrm{R} 3 \& \mathrm{R} 1+ \\
\gamma(\mathrm{CCO}) \mathrm{R} 4 \& \mathrm{R} 1+\gamma(\mathrm{CCC}) \mathrm{R} 4, \mathrm{R} 3 \& \mathrm{R} 1\end{array}$ \\
\hline 28 & 447 & 447 & & & & 5 & $\begin{array}{l}\beta(\mathrm{CNC})+\beta(\mathrm{CCC}) \mathrm{R} 1 \& \mathrm{R} 3+ \\
\beta(\mathrm{COC}) \mathrm{R} 4\end{array}$ \\
\hline 29 & 461 & 461 & & & & 5 & $\gamma(\mathrm{CCC}) \mathrm{R} 4+\gamma(\mathrm{CCO}) \mathrm{R} 4$ \\
\hline 30 & 475 & 476 & & & & 6 & $\begin{array}{l}\gamma(\mathrm{CCC}) \mathrm{R} 1, \mathrm{R} 2, \mathrm{R} 3 \& \mathrm{R} 4+ \\
\beta(\mathrm{COC}) \mathrm{R} 1+\gamma(\mathrm{CNC})+ \\
\gamma(\mathrm{CCO}) \mathrm{R} 1+\gamma(\mathrm{CCN})\end{array}$ \\
\hline 31 & 500 & 501 & & & & 102 & $\gamma(\mathrm{OH}) \mathrm{R} 4$ \\
\hline 32 & 506 & 507 & 514 & 506 & 508 & 8 & $\begin{array}{l}\gamma(\mathrm{OH}) \mathrm{R} 4+\gamma(\mathrm{CCC}) \mathrm{R} 1, \mathrm{R} 2, \mathrm{R} 3 \& \mathrm{R} 4+ \\
\beta(\mathrm{CNC})+\beta(\mathrm{CCN})\end{array}$ \\
\hline 33 & 521 & 522 & & & & 0 & $\begin{array}{l}\beta(\mathrm{CCC}) \mathrm{R} 4, \mathrm{R} 2 \& \mathrm{R} 1+\beta(\mathrm{COC}) \mathrm{R} 4+ \\
\beta(\mathrm{CCN})+\beta(\mathrm{CNC})\end{array}$ \\
\hline 34 & 533 & 534 & 530 & & & 9 & $\begin{array}{l}\gamma(\mathrm{COC}) \mathrm{R} 4 \& \mathrm{R} 1+ \\
\beta(\mathrm{CCC}) \mathrm{R} 1, \mathrm{R} 2, \mathrm{R} 3 \& \mathrm{R} 4+\beta(\mathrm{CCN})\end{array}$ \\
\hline 35 & 558 & 559 & 560 & & & 4 & $\gamma(\mathrm{CCC}) \mathrm{R} 1, \mathrm{R} 2, \mathrm{R} 3 \& \mathrm{R} 4+\gamma(\mathrm{CCO}) \mathrm{R} 1$ \\
\hline 36 & 565 & 566 & & & & 1 & $\begin{array}{l}\gamma(\mathrm{CCC}) \mathrm{R} 1, \mathrm{R} 2, \mathrm{R} 3 \& \mathrm{R} 4+\gamma(\mathrm{CNC})+ \\
\gamma(\mathrm{CCN})\end{array}$ \\
\hline 37 & 615 & 616 & 620 & & & 18 & $\begin{array}{l}\beta(\mathrm{CCC}) \mathrm{R} 3 \& \mathrm{R} 4+\gamma(\mathrm{CCC}) \mathrm{R} 1 \& \mathrm{R} 2+ \\
\gamma(\mathrm{CCO}) \mathrm{R} 1+\nu(\mathrm{CN})\end{array}$ \\
\hline 38 & 632 & 633 & & & & 1 & $\beta(\mathrm{CCC}) \mathrm{R} 2 \& \mathrm{R} 4+\beta(\mathrm{CCO}) \mathrm{R} 4$ \\
\hline 39 & 661 & 662 & & & & 6 & $\gamma(\mathrm{CCC}) \mathrm{R} 4, \mathrm{R} 3 \& \mathrm{R} 1+\gamma(\mathrm{CCO}) \mathrm{R} 4 \& \mathrm{R} 1$ \\
\hline 40 & 684 & 685 & 665 & 671 & 671 & 7 & $\begin{array}{l}\gamma(\mathrm{CCC}) \mathrm{R} 1, \mathrm{R} 2, \mathrm{R} 3 \& \mathrm{R} 4+ \\
\gamma(\mathrm{CCO}) \mathrm{R} 4 \& \mathrm{R} 1+\gamma(\mathrm{CCN})\end{array}$ \\
\hline 41 & 688 & 689 & & & & 1 & $\begin{array}{l}\gamma(\mathrm{CCC}) \mathrm{R} 1, \mathrm{R} 2, \mathrm{R} 3 \& \mathrm{R} 4+ \\
\gamma(\mathrm{CCO}) \mathrm{R} 4 \& \mathrm{R} 1+\beta(\mathrm{COC}) \mathrm{R} 1+ \\
\rho\left(\mathrm{CH}_{2}\right) \mathrm{R} 2 \& \mathrm{R} 3\end{array}$ \\
\hline 42 & 713 & 714 & & & & 2 & $\begin{array}{l}\gamma(\mathrm{CCC}) \mathrm{R} 4, \mathrm{R} 3 \& \mathrm{R} 1+ \\
\gamma(\mathrm{CCO}) \mathrm{R} 4 \& \mathrm{R} 1+\beta(\mathrm{COC}) \mathrm{R} 1\end{array}$ \\
\hline 43 & 727 & 728 & & & & 2 & $\begin{array}{l}\beta(\mathrm{CCC}) \mathrm{R} 1 \& \mathrm{R} 3+\beta(\mathrm{CCN})+ \\
\nu(\mathrm{CN})+\nu(\mathrm{CO}) \mathrm{R} 1\end{array}$ \\
\hline 44 & 760 & 761 & & & & 3 & $\begin{array}{l}\gamma(\mathrm{CCC}) \mathrm{R} 1+\beta(\mathrm{CCC}) \mathrm{R} 3 \& \mathrm{R} 4+ \\
\gamma(\mathrm{CCO}) \mathrm{R} 1+\nu(\mathrm{CN})\end{array}$ \\
\hline 45 & 762 & 763 & & & & 4 & $\gamma(\mathrm{CCC}) \mathrm{R} 1, \mathrm{R} 3 \& \mathrm{R} 4+\gamma(\mathrm{CCO}) \mathrm{R} 1$ \\
\hline 46 & 788 & 789 & 780 & 763 & 762 & 29 & $\begin{array}{l}\gamma(\mathrm{CCC}) \mathrm{R} 1+\beta(\mathrm{CCC}) \mathrm{R} 4+ \\
\nu(\mathrm{CC}) \mathrm{R} 2 \& \mathrm{R} 4+\beta(\mathrm{COC}) \mathrm{R} 1 \& \mathrm{R} 4\end{array}$ \\
\hline 47 & 822 & 790 & & & & 16 & $\begin{array}{l}\gamma(\mathrm{CH}) \mathrm{R} 1+\rho\left(\mathrm{CH}_{2}\right) \mathrm{R} 2+ \\
\gamma(\mathrm{CCC}) \mathrm{R} 1 \& \mathrm{R} 3+\nu(\mathrm{CN})+\beta(\mathrm{CNC})\end{array}$ \\
\hline
\end{tabular}


Table 2

(Continued)

\begin{tabular}{|c|c|c|c|c|c|c|c|}
\hline \multirow[t]{2}{*}{ S. No. } & \multicolumn{2}{|c|}{ Wave number } & \multirow{2}{*}{$\begin{array}{l}\text { Exp. IR freq. } \\
\text { in solid phase }\end{array}$} & \multirow{2}{*}{$\begin{array}{l}\text { Exp. IR freq. } \\
(5 \mathrm{mg} / \mathrm{ml}) \text { in } \\
\mathrm{CHCl}_{3}\end{array}$} & \multirow{2}{*}{$\begin{array}{l}\text { Exp. IR freq. } \\
(10 \mathrm{mg} / \mathrm{ml}) \text { in } \\
\mathrm{CHCl}_{3}\end{array}$} & \multirow{2}{*}{$\begin{array}{c}\text { IR } \\
\text { intensity }\end{array}$} & \multirow[t]{2}{*}{ Assignment } \\
\hline & Unscal. & Scal. & & & & & \\
\hline$\overline{48}$ & 832 & 799 & & & & 10 & $\begin{array}{l}\gamma(\mathrm{CH}) \mathrm{R} 1+\beta(\mathrm{CCC}) \mathrm{R} 3+\nu(\mathrm{CN})+ \\
\beta(\mathrm{CNC})+\gamma(\mathrm{CCC}) \mathrm{R} 1+\nu(\mathrm{CO}) \mathrm{R} 4+ \\
\nu(\mathrm{CC}) \mathrm{R} 4\end{array}$ \\
\hline 49 & 851 & 818 & & & & 13 & $\begin{array}{l}\nu(\mathrm{CN})+\rho\left(\mathrm{CH}_{2}\right) \mathrm{R} 2+\gamma(\mathrm{CH}) \mathrm{R} 1+ \\
\beta(\mathrm{CCN})\end{array}$ \\
\hline 50 & 874 & 840 & 850 & & 878 & 7 & $\gamma(\mathrm{CH}) \mathrm{R} 4+\gamma(\mathrm{CCC}) \mathrm{R} 4$ \\
\hline 51 & 894 & 859 & & & & 10 & $\begin{array}{l}\beta(\mathrm{CCC}) \mathrm{R} 1+\nu(\mathrm{CC}) \mathrm{R} 2, \mathrm{R} 3 \& \mathrm{R} 4+ \\
\beta(\mathrm{CNC})+\beta(\mathrm{CCN})+\nu(\mathrm{CO}) \mathrm{R} 1 \& \mathrm{R} 4\end{array}$ \\
\hline 52 & 899 & 864 & & & & 33 & $\gamma(\mathrm{CH}) \mathrm{R} 4$ \\
\hline 53 & 942 & 905 & & & & 51 & $\nu(\mathrm{CN})+\beta(\mathrm{CCN})+\beta(\mathrm{CCC}) \mathrm{R} 2 \& \mathrm{R} 3$ \\
\hline 54 & 949 & 912 & & & & 7 & $\begin{array}{l}\nu(\mathrm{CN})+\beta(\mathrm{CCN})+ \\
\beta(\mathrm{CCC}) \mathrm{R} 1 \& \mathrm{R} 2+\rho\left(\mathrm{CH}_{3}\right) \mathrm{R} 2+ \\
\beta(\mathrm{CNC})\end{array}$ \\
\hline 55 & 990 & 952 & 915 & 930 & 929 & 6 & $\begin{array}{l}\rho\left(\mathrm{CH}_{2}\right) \mathrm{R} 3+\beta(\mathrm{CCC}) \mathrm{R} 1, \mathrm{R} 2 \& \mathrm{R} 3+ \\
\nu(\mathrm{CN})+\nu(\mathrm{CO}) \mathrm{R} 1+ \\
\nu(\mathrm{CC}) \mathrm{R} 1, \mathrm{R} 2, \mathrm{R} 3 \& \mathrm{R} 4\end{array}$ \\
\hline 56 & 1014 & 975 & & & & 45 & $\begin{array}{l}\nu(\mathrm{CO}) \mathrm{R} 1+ \\
\nu(\mathrm{CC}) \mathrm{R} 2+\nu(\mathrm{CN})+ \\
\beta(\mathrm{CCC}) \mathrm{R} 1 \& \mathrm{R} 2+\beta(\mathrm{CCN})\end{array}$ \\
\hline 57 & 1023 & 983 & & & & 2 & $\begin{array}{l}\nu(\mathrm{CN})+\nu(\mathrm{CC}) \mathrm{R} 2 \& \mathrm{R} 3+ \\
\beta(\mathrm{CCC}) \mathrm{R} 1, \mathrm{R} 3 \& \mathrm{R} 4+ \\
\rho\left(\mathrm{CH}_{2}\right) \mathrm{R} 2 \& \mathrm{R} 3+\nu(\mathrm{CO}) \mathrm{R} 1\end{array}$ \\
\hline 58 & 1045 & 1004 & & & & 27 & $\begin{array}{l}\nu(\mathrm{CO}) \mathrm{R} 4+\nu(\mathrm{CN})+ \\
\nu(\mathrm{CC}) \mathrm{R} 1, \mathrm{R} 3 \& \mathrm{R} 4+\rho\left(\mathrm{CH}_{2}\right) \mathrm{R} 2+ \\
\beta(\mathrm{CCC}) \mathrm{R} 4\end{array}$ \\
\hline 59 & 1055 & 1014 & 1015 & 1003 & 1003 & 42 & $\begin{array}{l}\nu(\mathrm{CO}) \mathrm{R} 4+\nu(\mathrm{CN})+ \\
\nu(\mathrm{CC}) \mathrm{R} 2, \mathrm{R} 3 \& \mathrm{R} 4+\beta(\mathrm{CCC}) \mathrm{R} 4\end{array}$ \\
\hline 60 & 1062 & 1021 & & & & 14 & $\begin{array}{l}\nu(\mathrm{CC}) \mathrm{R} 2 \& \mathrm{R} 3+\nu(\mathrm{CN})+ \\
\nu(\mathrm{CO}) \mathrm{R} 1+\beta(\mathrm{CCC}) \mathrm{R} 1, \mathrm{R} 2 \& \mathrm{R} 3\end{array}$ \\
\hline 61 & 1085 & 1043 & 1035 & & & 44 & $\begin{array}{l}\nu(\mathrm{CC}) \mathrm{R} 2 \& \mathrm{R} 3+\nu(\mathrm{CN})+ \\
\beta(\mathrm{CCC}) \mathrm{R} 1 \& \mathrm{R} 4+\nu(\mathrm{CO}) \mathrm{R} 1 \& \mathrm{R} 4\end{array}$ \\
\hline 62 & 1121 & 1078 & 1080 & 1087 & 1086 & 142 & $\begin{array}{l}\nu(\mathrm{CO}) \mathrm{R} 1 \& \mathrm{R} 4+ \\
\beta(\mathrm{CCC}) \mathrm{R} 4 \& \mathrm{R} 1+\nu(\mathrm{CC}) \mathrm{R} 3+ \\
\beta(\mathrm{OH}) \mathrm{R} 1 \& \mathrm{R} 4\end{array}$ \\
\hline 63 & 1144 & 1100 & & & & 4 & $\begin{array}{l}\rho\left(\mathrm{CH}_{3}\right) \mathrm{R} 2+\mathrm{t}\left(\mathrm{CH}_{2}\right) \mathrm{R} 2+ \\
\nu(\mathrm{CC}) \mathrm{R} 2 \& \mathrm{R} 3+\nu(\mathrm{CO}) \mathrm{R} 1+ \\
\beta(\mathrm{CCC}) \mathrm{R} 1+\beta(\mathrm{CH}) \mathrm{R} 2 \& \mathrm{R} 3+ \\
\beta(\mathrm{CNC})\end{array}$ \\
\hline 64 & 1151 & 1106 & & & & 12 & $\begin{array}{l}\nu(\mathrm{CN})+\nu(\mathrm{CC}) \mathrm{R} 2 \& \mathrm{R} 3+ \\
\nu(\mathrm{CO}) \mathrm{R} 1 \& \mathrm{R} 4+\beta(\mathrm{CCC}) \mathrm{R} 1 \& \mathrm{R} 4\end{array}$ \\
\hline 65 & 1177 & 1131 & & & & 1 & $\begin{array}{l}\nu(\mathrm{CN})+\rho\left(\mathrm{CH}_{3}\right) \mathrm{R} 2 \& \mathrm{R} 1+ \\
\mathrm{t}\left(\mathrm{CH}_{2}\right) \mathrm{R} 2+\beta(\mathrm{CH}) \mathrm{R} 2 \& \mathrm{R} 3\end{array}$ \\
\hline 66 & 1179 & 1133 & & & & 5 & $\rho\left(\mathrm{CH}_{3}\right) \mathrm{R} 1$ \\
\hline 67 & 1183 & 1137 & & & & 1 & $\rho\left(\mathrm{CH}_{3}\right) \mathrm{R} 4$ \\
\hline
\end{tabular}


Table 2

(Continued)

\begin{tabular}{|c|c|c|c|c|c|c|c|}
\hline \multirow[t]{2}{*}{ S. No. } & \multicolumn{2}{|c|}{ Wave number } & \multirow{2}{*}{$\begin{array}{l}\text { Exp. IR freq. } \\
\text { in solid phase }\end{array}$} & \multirow{2}{*}{$\begin{array}{l}\text { Exp. IR freq. } \\
(5 \mathrm{mg} / \mathrm{ml}) \text { in } \\
\mathrm{CHCl}_{3}\end{array}$} & \multirow{2}{*}{$\begin{array}{l}\text { Exp. IR freq. } \\
(10 \mathrm{mg} / \mathrm{ml}) \text { in } \\
\mathrm{CHCl}_{3}\end{array}$} & \multirow{2}{*}{$\begin{array}{c}\text { IR } \\
\text { intensity }\end{array}$} & \multirow[t]{2}{*}{ Assignment } \\
\hline & Unscal. & Scal. & & & & & \\
\hline 68 & 1191 & 1145 & 1142 & & & 7 & $\begin{array}{l}\nu(\mathrm{CC}) \mathrm{R} 1, \mathrm{R} 2, \mathrm{R} 3 \& \mathrm{R} 4+ \\
\beta(\mathrm{CH}) \mathrm{R} 1, \mathrm{R} 2, \mathrm{R} 3 \& \mathrm{R} 4+ \\
\beta(\mathrm{OH}) \mathrm{R} 1+\rho\left(\mathrm{CH}_{3}\right) \mathrm{R} 4 \& \mathrm{R} 1+ \\
\nu(\mathrm{CO}) \mathrm{R} 4\end{array}$ \\
\hline 69 & 1203 & 1157 & & & & 16 & $\begin{array}{l}\mathrm{t}\left(\mathrm{CH}_{2}\right) \mathrm{R} 3 \& \mathrm{R} 2+\beta(\mathrm{CH}) \mathrm{R} 2 \& \mathrm{R} 3+ \\
\beta(\mathrm{NH})+\rho\left(\mathrm{CH}_{3}\right) \mathrm{R} 1 \& \mathrm{R} 2+ \\
\beta(\mathrm{OH}) \mathrm{R} 1+\nu(\mathrm{CC}) \mathrm{R} 4\end{array}$ \\
\hline 70 & 1211 & 1164 & & & & 40 & $\begin{array}{l}\rho\left(\mathrm{CH}_{3}\right) \mathrm{R} 4+\beta(\mathrm{CH}) \mathrm{R} 4+\beta(\mathrm{OH}) \mathrm{R} 4+ \\
\nu(\mathrm{CC}) \mathrm{R} 4+\nu(\mathrm{CO}) \mathrm{R} 4+\beta(\mathrm{CCC}) \mathrm{R} 4\end{array}$ \\
\hline 71 & 1218 & 1170 & & & & 43 & $\begin{array}{l}\rho\left(\mathrm{CH}_{3}\right) \mathrm{R} 1+\beta(\mathrm{OH}) \mathrm{R} 1+ \\
\nu(\mathrm{CC}) \mathrm{R} 1 \& \mathrm{R} 3+\beta(\mathrm{COC}) \mathrm{R} 1\end{array}$ \\
\hline 72 & 1226 & 1179 & & & & 12 & $\begin{array}{l}\beta(\mathrm{CH}) \mathrm{R} 1 \& \mathrm{R} 4+\beta(\mathrm{OH}) \mathrm{R} 1+ \\
\nu(\mathrm{CC}) \mathrm{R} 1 \& \mathrm{R} 3+\rho\left(\mathrm{CH}_{3}\right) \mathrm{R} 1, \mathrm{R} 2 \& \mathrm{R} 4+ \\
\nu(\mathrm{CO}) \mathrm{R} 4\end{array}$ \\
\hline 73 & 1236 & 1188 & 1185 & & & 84 & $\begin{array}{l}\nu(\mathrm{CO}) \mathrm{R} 4+\rho\left(\mathrm{CH}_{3}\right) \mathrm{R} 1 \& \mathrm{R} 4+ \\
\nu(\mathrm{CC}) \mathrm{R} 4 \& \mathrm{R} 3+\beta(\mathrm{OH}) \mathrm{R} 1 \& \mathrm{R} 4+ \\
\mathrm{t}\left(\mathrm{CH}_{2}\right) \mathrm{R} 2+\beta(\mathrm{NH})+ \\
\beta(\mathrm{CH}) \mathrm{R} 2 \& \mathrm{R} 3+\nu(\mathrm{CN})+\beta(\mathrm{CCC}) \mathrm{R} 4\end{array}$ \\
\hline 74 & 1247 & 1199 & & & & 24 & $\begin{array}{l}\beta(\mathrm{CH}) \mathrm{R} 1, \mathrm{R} 2, \mathrm{R} 3 \& \mathrm{R} 4+ \\
\mathrm{t}\left(\mathrm{CH}_{2}\right) \mathrm{R} 2 \& \mathrm{R} 3+\beta(\mathrm{OH}) \mathrm{R} 4+ \\
\rho\left(\mathrm{CH}_{3}\right) \mathrm{R} 1 \& \mathrm{R} 4+\beta(\mathrm{NH})+ \\
\nu(\mathrm{CC}) \mathrm{R} 1, \mathrm{R} 2, \mathrm{R} 3 \& \mathrm{R} 4\end{array}$ \\
\hline 75 & 1250 & 1201 & & & & 9 & $\begin{array}{l}\nu(\mathrm{CC}) \mathrm{R} 1, \mathrm{R} 3 \& \mathrm{R} 4+ \\
\beta(\mathrm{CH}) \mathrm{R} 1, \mathrm{R} 2, \mathrm{R} 3 \& \mathrm{R} 4+ \\
\beta(\mathrm{OH}) \mathrm{R} 1 \& \mathrm{R} 4+\nu(\mathrm{CO}) \mathrm{R} 1+ \\
\rho\left(\mathrm{CH}_{3}\right) \mathrm{R} 1 \& \mathrm{R} 4\end{array}$ \\
\hline 76 & 1278 & 1228 & 1213 & 1216 & 1216 & 73 & $\begin{array}{l}\beta(\mathrm{CH}) \mathrm{R} 4+\mathrm{t}\left(\mathrm{CH}_{2}\right) \mathrm{R} 2 \& \mathrm{R} 3+ \\
\nu(\mathrm{CO}) \mathrm{R} 4+\beta(\mathrm{OH}) \mathrm{R} 4+\beta(\mathrm{CCC}) \mathrm{R} 4+ \\
\nu(\mathrm{CC}) \mathrm{R} 3 \& \mathrm{R} 4\end{array}$ \\
\hline 77 & 1290 & 1240 & & & & 167 & $\begin{array}{l}\nu(\mathrm{CO}) \mathrm{R} 1+\beta(\mathrm{CH}) \mathrm{R} 1, \mathrm{R} 2, \mathrm{R} 3 \& \mathrm{R} 4+ \\
\nu(\mathrm{CC}) \mathrm{R} 1 \& \mathrm{R} 3+\beta(\mathrm{CCC}) \mathrm{R} 1+ \\
\beta(\mathrm{OH}) \mathrm{R} 1 \& \mathrm{R} 4+t\left(\mathrm{CH}_{2}\right) \mathrm{R} 2+ \\
\omega\left(\mathrm{CH}_{3}\right) \mathrm{R} 1\end{array}$ \\
\hline 78 & 1291 & 1241 & 1245 & & & 179 & $\begin{array}{l}\mathrm{t}\left(\mathrm{CH}_{2}\right) \mathrm{R} 2+\nu(\mathrm{CO}) \mathrm{R} 4 \& \mathrm{R} 1+ \\
\nu(\mathrm{CC}) \mathrm{R} 1, \mathrm{R} 2, \mathrm{R} 3 \& \mathrm{R} 4+ \\
\beta(\mathrm{CH}) \mathrm{R} 1, \mathrm{R} 2 \& \mathrm{R} 3+\beta(\mathrm{OH}) \mathrm{R} 1 \& \mathrm{R} 4\end{array}$ \\
\hline 79 & 1306 & 1256 & & & & 41 & $\begin{array}{l}\nu(\mathrm{CC}) \mathrm{R} 1, \mathrm{R} 2, \mathrm{R} 3 \& \mathrm{R} 4+ \\
\nu(\mathrm{CO}) \mathrm{R} 4 \& \mathrm{R} 1+\beta(\mathrm{CH}) \mathrm{R} 1 \& \mathrm{R} 4+ \\
\beta(\mathrm{OH}) \mathrm{R} 4+\mathrm{t}\left(\mathrm{CH}_{2}\right) \mathrm{R} 2\end{array}$ \\
\hline 80 & 1330 & 1278 & & & & 78 & $\begin{array}{l}\omega\left(\mathrm{CH}_{2}\right) \mathrm{R} 3 \& \mathrm{R} 2+\nu(\mathrm{CO}) \mathrm{R} 4 \& \mathrm{R} 1+ \\
\beta(\mathrm{CH}) \mathrm{R} 2, \mathrm{R} 3 \& \mathrm{R} 4+ \\
\nu(\mathrm{CC}) \mathrm{R} 1, \mathrm{R} 2, \mathrm{R} 3 \& \mathrm{R} 4+\mathrm{t}\left(\mathrm{CH}_{2}\right) \mathrm{R} 2+ \\
\beta(\mathrm{OH}) \mathrm{R} 4\end{array}$ \\
\hline
\end{tabular}


Table 2

(Continued)

\begin{tabular}{|c|c|c|c|c|c|c|c|}
\hline \multirow[t]{2}{*}{ S. No. } & \multicolumn{2}{|c|}{ Wave number } & \multirow{2}{*}{$\begin{array}{l}\text { Exp. IR freq. } \\
\text { in solid phase }\end{array}$} & \multirow{2}{*}{$\begin{array}{l}\text { Exp. IR freq. } \\
(5 \mathrm{mg} / \mathrm{ml}) \text { in } \\
\mathrm{CHCl}_{3}\end{array}$} & \multirow{2}{*}{$\begin{array}{l}\text { Exp. IR freq. } \\
(10 \mathrm{mg} / \mathrm{ml}) \text { in } \\
\mathrm{CHCl}_{3}\end{array}$} & \multirow{2}{*}{$\begin{array}{c}\text { IR } \\
\text { intensity }\end{array}$} & \multirow[t]{2}{*}{ Assignment } \\
\hline & Unscal. & Scal. & & & & & \\
\hline 81 & 1339 & 1287 & & & & 54 & $\begin{array}{l}\beta(\mathrm{CH}) \mathrm{R} 2, \mathrm{R} 3 \& \mathrm{R} 4+\beta(\mathrm{OH}) \mathrm{R} 1 \& \mathrm{R} 4+ \\
\nu(\mathrm{CO}) \mathrm{R} 1 \& \mathrm{R} 4+\nu(\mathrm{CC}) \mathrm{R} 1, \mathrm{R} 3 \& \mathrm{R} 4+ \\
\beta(\mathrm{CCC}) \mathrm{R} 1 \& \mathrm{R} 4+\omega\left(\mathrm{CH}_{2}\right) \mathrm{R} 2\end{array}$ \\
\hline 82 & 1352 & 1299 & 1295 & & & 68 & $\begin{array}{l}\nu(\mathrm{CC}) \mathrm{R} 1, \mathrm{R} 2, \mathrm{R} 3 \& \mathrm{R} 4+ \\
\beta(\mathrm{CH}) \mathrm{R} 2, \mathrm{R} 3 \& \mathrm{R} 4+\mathrm{t}\left(\mathrm{CH}_{2}\right) \mathrm{R} 2+ \\
\nu(\mathrm{CO}) \mathrm{R} 1 \& \mathrm{R} 4+\beta(\mathrm{OH}) \mathrm{R} 1 \& \mathrm{R} 4\end{array}$ \\
\hline 83 & 1365 & 1312 & & & & 77 & $\begin{array}{l}\beta(\mathrm{CH}) \mathrm{R} 2 \& \mathrm{R} 3+\nu(\mathrm{CC}) \mathrm{R} 1, \mathrm{R} 3 \& \mathrm{R} 4+ \\
\nu(\mathrm{CO}) \mathrm{R} 1 \& \mathrm{R} 4+\beta(\mathrm{NH})\end{array}$ \\
\hline 84 & 1375 & 1322 & & & & 24 & $\begin{array}{l}\beta(\mathrm{CH}) \mathrm{R} 2 \& \mathrm{R} 3+ \\
\nu(\mathrm{CC}) \mathrm{R} 1, \mathrm{R} 2, \mathrm{R} 3 \& \mathrm{R} 4+\nu(\mathrm{CO}) \mathrm{R} 1 \& \mathrm{R} 4\end{array}$ \\
\hline 85 & 1389 & 1336 & 1340 & & & 51 & $\begin{array}{l}\omega\left(\mathrm{CH}_{2}\right) \mathrm{R} 2+\nu(\mathrm{CC}) \mathrm{R} 1, \mathrm{R} 2, \mathrm{R} 3 \& \mathrm{R} 4+ \\
\mathrm{t}\left(\mathrm{CH}_{2}\right) \mathrm{R} 2+\beta(\mathrm{NH})+\nu(\mathrm{CO}) \mathrm{R} 1 \& \mathrm{R} 4+ \\
\beta(\mathrm{OH}) \mathrm{R} 1+\beta(\mathrm{CH}) \mathrm{R} 2 \& \mathrm{R} 3\end{array}$ \\
\hline 86 & 1399 & 1345 & & & & 55 & $\begin{array}{l}\omega\left(\mathrm{CH}_{2}\right) \mathrm{R} 2 \& \mathrm{R} 3+\beta(\mathrm{NH})+ \\
\nu(\mathrm{CC}) \mathrm{R} 1, \mathrm{R} 2, \mathrm{R} 3 \& \mathrm{R} 4+\omega\left(\mathrm{CH}_{3}\right) \mathrm{R} 2+ \\
\nu(\mathrm{CO}) \mathrm{R} 4 \& \mathrm{R} 1\end{array}$ \\
\hline 87 & 1407 & 1353 & & & & 98 & $\begin{array}{l}\nu(\mathrm{CC}) \mathrm{R} 1, \mathrm{R} 2, \mathrm{R} 3 \& \mathrm{R} 4+ \\
\mathrm{s}\left(\mathrm{CH}_{2}\right) \mathrm{R} 2 \& \mathrm{R} 3+\nu(\mathrm{CO}) \mathrm{R} 4 \& \mathrm{R} 1+ \\
\beta(\mathrm{NH})+\beta(\mathrm{OH}) \mathrm{R} 4 \& \mathrm{R} 1+ \\
\beta(\mathrm{CH}) \mathrm{R} 2, \mathrm{R} 3 \& \mathrm{R} 4+\omega\left(\mathrm{CH}_{3}\right) \mathrm{R} 2\end{array}$ \\
\hline 88 & 1424 & 1369 & & & & 20 & $\begin{array}{l}\beta(\mathrm{NH})+\nu(\mathrm{CC}) \mathrm{R} 4, \mathrm{R} 3 \& \mathrm{R} 1+ \\
\beta(\mathrm{OH}) \mathrm{R} 4 \& \mathrm{R} 1+\beta(\mathrm{CH}) \mathrm{R} 2 \& \mathrm{R} 3+ \\
\nu(\mathrm{CO}) \mathrm{R} 4+\nu(\mathrm{CN})+ \\
\omega\left(\mathrm{CH}_{3}\right) \mathrm{R} 2 \& \mathrm{R} 4+\omega\left(\mathrm{CH}_{2}\right) \mathrm{R} 2\end{array}$ \\
\hline 89 & 1429 & 1374 & & & & 81 & $\begin{array}{l}\beta(\mathrm{NH})+\beta(\mathrm{CH}) \mathrm{R} 2 \& \mathrm{R} 3+ \\
\mathrm{t}\left(\mathrm{CH}_{2}\right) \mathrm{R} 2+\nu(\mathrm{CC}) \mathrm{R} 4 \& \mathrm{R} 3+ \\
\beta(\mathrm{OH}) \mathrm{R} 4+\nu(\mathrm{CN})+\nu(\mathrm{CO}) \mathrm{R} 4\end{array}$ \\
\hline 90 & 1448 & 1392 & 1385 & 1372 & 1373 & 63 & $\begin{array}{l}\beta(\mathrm{NH})+\omega\left(\mathrm{CH}_{2}\right) \mathrm{R} 2+\omega\left(\mathrm{CH}_{3}\right) \mathrm{R} 2+ \\
\nu(\mathrm{CN})+\beta(\mathrm{CH}) \mathrm{R} 2 \& \mathrm{R} 3\end{array}$ \\
\hline 91 & 1456 & 1400 & & & & 69 & $\begin{array}{l}\nu(\mathrm{CC}) \mathrm{R} 1, \mathrm{R} 2, \mathrm{R} 3 \& \mathrm{R} 4+ \\
\nu(\mathrm{CO}) \mathrm{R} 1 \& \mathrm{R} 4+\omega\left(\mathrm{CH}_{2}\right) \mathrm{R} 2+ \\
\omega\left(\mathrm{CH}_{3}\right) \mathrm{R} 2+\beta(\mathrm{OH}) \mathrm{R} 4+ \\
\beta(\mathrm{CH}) \mathrm{R} 2 \& \mathrm{R} 3\end{array}$ \\
\hline 92 & 1479 & 1422 & & & & 8 & $\begin{array}{l}\omega\left(\mathrm{CH}_{3}\right) \mathrm{R} 2+\omega\left(\mathrm{CH}_{2}\right) \mathrm{R} 2+ \\
\beta(\mathrm{CH}) \mathrm{R} 2 \& \mathrm{R} 3\end{array}$ \\
\hline 93 & 1482 & 1425 & & & & 41 & $\begin{array}{l}\nu(\mathrm{CC}) \mathrm{R} 1, \mathrm{R} 3 \& \mathrm{R} 4+ \\
\omega\left(\mathrm{CH}_{3}\right) \mathrm{R} 1 \& \mathrm{R} 4+\beta(\mathrm{OH}) \mathrm{R} 1 \& \mathrm{R} 4+ \\
\nu(\mathrm{CO}) \mathrm{R} 4+\beta(\mathrm{CH}) \mathrm{R} 4\end{array}$ \\
\hline 94 & 1496 & 1438 & & & & 12 & $\begin{array}{l}\omega\left(\mathrm{CH}_{3}\right) \mathrm{R} 1 \& \mathrm{R} 4+ \\
\nu(\mathrm{CC}) \mathrm{R} 1, \mathrm{R} 3 \& \mathrm{R} 4+\mathrm{s}\left(\mathrm{CH}_{2}\right) \mathrm{R} 3+ \\
\beta(\mathrm{OH}) \mathrm{R} 4 \& \mathrm{R} 1+\nu(\mathrm{CO}) \mathrm{R} 4 \& \mathrm{R} 1\end{array}$ \\
\hline 95 & 1506 & 1447 & & & & 12 & $\begin{array}{l}\omega\left(\mathrm{CH}_{3}\right) \mathrm{R} 4+ \\
\mathrm{s}\left(\mathrm{CH}_{2}\right) \mathrm{R} 3 \& \mathrm{R} 2+\mathrm{s}\left(\mathrm{CH}_{3}\right) \mathrm{R} 2+ \\
\nu(\mathrm{CC}) \mathrm{R} 3 \& \mathrm{R} 4+\nu(\mathrm{CO}) \mathrm{R} 4 \\
\end{array}$ \\
\hline
\end{tabular}


Table 2

(Continued)

\begin{tabular}{|c|c|c|c|c|c|c|c|}
\hline \multirow[t]{2}{*}{ S. No. } & \multicolumn{2}{|c|}{ Wave number } & \multirow{2}{*}{$\begin{array}{l}\text { Exp. IR freq. } \\
\text { in solid phase }\end{array}$} & \multirow{2}{*}{$\begin{array}{l}\text { Exp. IR freq. } \\
(5 \mathrm{mg} / \mathrm{ml}) \text { in } \\
\mathrm{CHCl}_{3}\end{array}$} & \multirow{2}{*}{$\begin{array}{l}\text { Exp. IR freq. } \\
(10 \mathrm{mg} / \mathrm{ml}) \text { in } \\
\mathrm{CHCl}_{3}\end{array}$} & \multirow{2}{*}{$\begin{array}{c}\text { IR } \\
\text { intensity }\end{array}$} & \multirow[t]{2}{*}{ Assignment } \\
\hline & Unscal. & Scal. & & & & & \\
\hline 96 & 1507 & 1449 & & & & 2 & $\begin{array}{l}\mathrm{s}\left(\mathrm{CH}_{2}\right) \mathrm{R} 3 \& \mathrm{R} 2+\omega\left(\mathrm{CH}_{3}\right) \mathrm{R} 4 \& \mathrm{R} 1+ \\
\mathrm{s}\left(\mathrm{CH}_{3}\right) \mathrm{R} 2+\nu(\mathrm{CC}) \mathrm{R} 3 \& \mathrm{R} 4\end{array}$ \\
\hline 97 & 1512 & 1453 & & & & 8 & $\mathrm{~s}\left(\mathrm{CH}_{2}\right) \mathrm{R} 2 \& \mathrm{R} 3+\mathrm{s}\left(\mathrm{CH}_{3}\right) \mathrm{R} 2$ \\
\hline 98 & 1516 & 1457 & & & & 6 & $\mathrm{~s}\left(\mathrm{CH}_{3}\right) \mathrm{R} 1, \mathrm{R} 2 \& \mathrm{R} 4$ \\
\hline 99 & 1517 & 1459 & & & & 2 & $\mathrm{~s}\left(\mathrm{CH}_{3}\right) \mathrm{R} 2 \& \mathrm{R} 1+\mathrm{s}\left(\mathrm{CH}_{2}\right) \mathrm{R} 2 \& \mathrm{R} 1$ \\
\hline 100 & 1519 & 1460 & & & & 9 & $\mathrm{~s}\left(\mathrm{CH}_{3}\right) \mathrm{R} 4 \& \mathrm{R} 1$ \\
\hline 101 & 1529 & 1470 & & & & 16 & $\mathrm{~s}\left(\mathrm{CH}_{2}\right) \mathrm{R} 2+\mathrm{s}\left(\mathrm{CH}_{3}\right) \mathrm{R} 2$ \\
\hline 102 & 1530 & 1471 & & & & 90 & $\mathrm{~s}\left(\mathrm{CH}_{3}\right) \mathrm{R} 4$ \\
\hline 103 & 1533 & 1474 & 1472 & 1468 & 1468 & 26 & $\mathrm{~s}\left(\mathrm{CH}_{3}\right) \mathrm{R} 2+\mathrm{s}\left(\mathrm{CH}_{2}\right) \mathrm{R} 2 \& \mathrm{R} 3+\beta(\mathrm{NH})$ \\
\hline 104 & 1534 & 1475 & & & & 12 & $\mathrm{~s}\left(\mathrm{CH}_{3}\right) \mathrm{R} 1$ \\
\hline 105 & 1540 & 1481 & & & & 89 & $\begin{array}{l}\nu(\mathrm{CO}) \mathrm{R} 1 \& \mathrm{R} 4+\nu(\mathrm{CC}) \mathrm{R} 1, \mathrm{R} 3 \& \mathrm{R} 4+ \\
\omega\left(\mathrm{CH}_{3}\right) \mathrm{R} 1+\mathrm{s}\left(\mathrm{CH}_{3}\right) \mathrm{R} 4+ \\
\beta(\mathrm{CH}) \mathrm{R} 1+\beta(\mathrm{OH}) \mathrm{R} 1 \& \mathrm{R} 4+ \\
\beta(\mathrm{CCC}) \mathrm{R} 3\end{array}$ \\
\hline 106 & 1570 & 1510 & 1505 & 1514 & 1513 & 199 & $\begin{array}{l}\nu(\mathrm{CO}) \mathrm{R} 4+\beta(\mathrm{CH}) \mathrm{R} 1 \& \mathrm{R} 4+ \\
\nu(\mathrm{CC}) \mathrm{R} 1 \& \mathrm{R} 4+\omega\left(\mathrm{CH}_{3}\right) \mathrm{R} 4\end{array}$ \\
\hline 107 & 1632 & 1568 & & & & 101 & $\begin{array}{l}\nu(\mathrm{CC}) \mathrm{R} 1 \& \mathrm{R} 4+\beta(\mathrm{OH}) \mathrm{R} 4 \& \mathrm{R} 1+ \\
\beta(\mathrm{CH}) \mathrm{R} 1 \& \mathrm{R} 3\end{array}$ \\
\hline 108 & 1636 & 1573 & & & & 95 & $\begin{array}{l}\nu(\mathrm{CC}) \mathrm{R} 1 \& \mathrm{R} 4+\nu(\mathrm{CO}) \mathrm{R} 1+ \\
\beta(\mathrm{CH}) \mathrm{R} 1+\beta(\mathrm{OH}) \mathrm{R} 1 \& \mathrm{R} 4\end{array}$ \\
\hline 109 & 1647 & 1583 & & & & 45 & $\begin{array}{l}\nu(\mathrm{CC}) \mathrm{R} 1 \& \mathrm{R} 4+\beta(\mathrm{OH}) \mathrm{R} 4 \& \mathrm{R} 1+ \\
\beta(\mathrm{CH}) \mathrm{R} 1+\nu(\mathrm{CO}) \mathrm{R} 1\end{array}$ \\
\hline 110 & 1669 & 1605 & 1590 & 1591 & 1593 & 34 & $\begin{array}{l}\nu(\mathrm{CC}) \mathrm{R} 4 \& \mathrm{R} 1+\nu(\mathrm{CO}) \mathrm{R} 4+ \\
\beta(\mathrm{CH}) \mathrm{R} 4+\beta(\mathrm{OH}) \mathrm{R} 4 \& \mathrm{R} 1\end{array}$ \\
\hline 111 & 3002 & 2886 & & & & 26 & $\nu_{\mathrm{s}}\left(\mathrm{CH}_{2}\right) \mathrm{R} 3$ \\
\hline 112 & 3027 & 2909 & & & & 19 & $\nu_{\mathrm{s}}\left(\mathrm{CH}_{2}\right) \mathrm{R} 2$ \\
\hline 113 & 3045 & 2927 & 2850 & 2847 & 2847 & 33 & $\nu_{\mathrm{s}}\left(\mathrm{CH}_{3}\right) \mathrm{R} 4$ \\
\hline 114 & 3046 & 2928 & & & & 13 & $\nu(\mathrm{CH}) \mathrm{R} 2 \& \mathrm{R} 3+\nu_{\mathrm{s}}\left(\mathrm{CH}_{2}\right) \mathrm{R} 3$ \\
\hline 115 & 3056 & 2938 & & & & 44 & $\nu_{\mathrm{s}}\left(\mathrm{CH}_{3}\right) \mathrm{R} 1$ \\
\hline 116 & 3093 & 2973 & 2925 & 2935 & 2960 & 2 & $\nu_{\mathrm{as}}\left(\mathrm{CH}_{2}\right) \mathrm{R} 2+\nu_{\mathrm{s}}\left(\mathrm{CH}_{2}\right) \mathrm{R} 2$ \\
\hline 117 & 3102 & 2982 & & & & 8 & $\begin{array}{l}\nu_{\mathrm{s}}\left(\mathrm{CH}_{2}\right) \mathrm{R} 2+\nu_{\mathrm{s}}\left(\mathrm{CH}_{3}\right) \mathrm{R} 2+ \\
\nu_{\mathrm{as}}\left(\mathrm{CH}_{2}\right) \mathrm{R} 2\end{array}$ \\
\hline 118 & 3106 & 2986 & & & & 2 & $\begin{array}{l}\nu_{\mathrm{s}}\left(\mathrm{CH}_{3}\right) \mathrm{R} 2+\nu_{\mathrm{s}}\left(\mathrm{CH}_{2}\right) \mathrm{R} 2+ \\
\nu_{\mathrm{as}}\left(\mathrm{CH}_{2}\right) \mathrm{R} 3\end{array}$ \\
\hline 119 & 3112 & 2992 & & & & 28 & $\nu_{\mathrm{as}}\left(\mathrm{CH}_{3}\right) \mathrm{R} 4$ \\
\hline 120 & 3115 & 2994 & & & & 25 & $\nu_{\mathrm{s}}\left(\mathrm{CH}_{3}\right) \mathrm{R} 2+\nu_{\mathrm{as}}\left(\mathrm{CH}_{2}\right) \mathrm{R} 3$ \\
\hline 121 & 3140 & 3019 & 3000 & & & 25 & $\nu_{\mathrm{as}}\left(\mathrm{CH}_{3}\right) \mathrm{R} 1$ \\
\hline 122 & 3157 & 3034 & 3020 & 3022 & 3022 & 6 & $\nu_{\text {as }}\left(\mathrm{CH}_{2}\right) \mathrm{R} 2$ \\
\hline 123 & 3175 & 3052 & & & & 42 & $\nu_{\mathrm{as}}\left(\mathrm{CH}_{3}\right) \mathrm{R} 1$ \\
\hline 124 & 3176 & 3053 & & & & 8 & $\nu(\mathrm{CH}) \mathrm{R} 1$ \\
\hline 125 & 3178 & 3055 & & & & 15 & $\nu_{\text {as }}\left(\mathrm{CH}_{3}\right) \mathrm{R} 4$ \\
\hline 126 & 3195 & 3071 & & & & 2 & $\nu_{\text {as }}\left(\mathrm{CH}_{3}\right) \mathrm{R} 2$ \\
\hline 127 & 3208 & 3083 & & & & 5 & $\nu(\mathrm{CH}) \mathrm{R} 4$ \\
\hline
\end{tabular}


Table 2

(Continued)

\begin{tabular}{|c|c|c|c|c|c|c|c|}
\hline \multirow[t]{2}{*}{ S. No. } & \multicolumn{2}{|c|}{ Wave number } & \multirow{2}{*}{$\begin{array}{l}\text { Exp. IR freq. } \\
\text { in solid phase }\end{array}$} & \multirow{2}{*}{$\begin{array}{l}\text { Exp. IR freq. } \\
(5 \mathrm{mg} / \mathrm{ml}) \text { in } \\
\mathrm{CHCl}_{3}\end{array}$} & \multirow{2}{*}{$\begin{array}{l}\text { Exp. IR freq. } \\
(10 \mathrm{mg} / \mathrm{ml}) \text { in } \\
\mathrm{CHCl}_{3}\end{array}$} & \multirow{2}{*}{$\begin{array}{c}\text { IR } \\
\text { intensity }\end{array}$} & \multirow[t]{2}{*}{ Assignment } \\
\hline & Unscal. & Scal. & & & & & \\
\hline 128 & 3220 & 3095 & & & & 1 & $\nu_{\mathrm{as}}\left(\mathrm{CH}_{3}\right) \mathrm{R} 2$ \\
\hline 129 & 3284 & 3157 & & & & 6 & $\nu(\mathrm{CH}) \mathrm{R} 4$ \\
\hline 130 & 3405 & 3273 & & & & 22 & $\nu(\mathrm{NH})$ \\
\hline 131 & 3688 & 3546 & 3420 & 3527 & 3430 & 151 & $\nu(\mathrm{OH}) \mathrm{R} 4$ \\
\hline 132 & 3747 & 3602 & 3680 & 3684 & 3682 & 90 & $\nu(\mathrm{OH}) \mathrm{R} 1$ \\
\hline
\end{tabular}

Notes: $\nu$ - stretching; $\nu_{\mathrm{s}}$ - symmetric stretching; $\nu_{\mathrm{as}}$ - asymmetric stretching; $\beta$ - in-plane-bending; $\gamma$ - out-of-plane bending; $\omega$ - wagging; $\rho$ - rocking; $\mathrm{t}$ - twisting; $\tau$ - torsion, $\mathrm{s}$ - scissoring.

$\mathrm{CH}_{2}$ asymmetric stretching vibrations are calculated at 3034 and $2973 \mathrm{~cm}^{-1}$, whereas $\mathrm{CH}_{2}$ symmetric stretching vibrations are calculated at 2982, 2909 and $2886 \mathrm{~cm}^{-1}$. The bands corresponding to different bending vibrations of $\mathrm{CH}_{2}$ group are summarized in Table 2 and are supported by the literature [15].

\section{5. $C-C$ vibrations}

The $\mathrm{C}-\mathrm{C}$ aromatic stretching band known as semi-circle stretching, calculated at 1583, 1299, 1145 and $1043 \mathrm{~cm}^{-1}$, respectively, are supported by observed frequencies in FT-IR spectra (solid phase), i.e. $1590,1295,1142$ and $1035 \mathrm{~cm}^{-1}$. Some of the other $\mathrm{C}-\mathrm{C}$ aromatic stretching band are also calculated at $1605,1573,1568,1425,1400,1353,1256,1201$ and $1021 \mathrm{~cm}^{-1}$, which are also supported by the literature [12]. The theoretically calculated $\mathrm{C}-\mathrm{C}-\mathrm{C}$ bending modes and $\mathrm{C}-\mathrm{C}$ torsional modes have been found to be consistent with the recorded spectral values and the literature [12].

\subsection{Methyl group vibrations}

The asymmetric $\mathrm{CH}_{3}$ stretching vibrations are calculated at 3095, 3071, 3055, 3052, 3019 and $2992 \mathrm{~cm}^{-1}$, while symmetric $\mathrm{CH}_{3}$ stretching vibrations are calculated at 2994, 2986, 2938 and $2927 \mathrm{~cm}^{-1}$. These assignments are supported by the experimental data and literature [3]. In the present study various bending vibrations of $\mathrm{CH}_{3}$ group are also summarized in Table 2 and are supported by the literature [3].

\section{7. $C-O$ vibrations}

In this study the $\mathrm{C}-\mathrm{O}$ stretching vibrations are calculated at $1510,1188,1078$ and $1014 \mathrm{~cm}^{-1}$, are supported by the observed frequencies in FT-IR spectra (solid phase), i.e. $1505,1185,1080$ and $1015 \mathrm{~cm}^{-1}$. Some of the other $\mathrm{C}-\mathrm{O}$ stretching vibrations are also calculated at 1481, 1240, 1004 and $975 \mathrm{~cm}^{-1}$, are also supported by the literature [12]. The various bending and torsional vibrations assigned in this study are also supported by the literature [12].

\section{8. $O-H$ vibrations}

In a vibrational spectra, the strength of hydrogen bond determines the position of $\mathrm{O}-\mathrm{H}$ band. Usually the O-H stretching appears at $3600-3400 \mathrm{~cm}^{-1}$ [13]. In this study boldine hydrochloride showed very strong absorption peaks at 3602 and $3546 \mathrm{~cm}^{-1}$, which are due to the $\mathrm{O}-\mathrm{H}$ stretching vibration and are 
supported by observed frequencies in FT-IR spectra (solid phase), i.e. 3680 and $3420 \mathrm{~cm}^{-1}$. The various bending vibrations of the hydroxyl groups are also found to be in good agreement with the observed spectra and literature [13].

\section{9. $N-H$ vibrations}

In all the heterocyclic compounds, the $\mathrm{N}-\mathrm{H}$ stretching vibrations [18] occurs in the region 3500$3000 \mathrm{~cm}^{-1}$. In the present study the $\mathrm{N}-\mathrm{H}$ vibration is calculated at $3273 \mathrm{~cm}^{-1}$, which is supported by the literature [18]. Some bending vibrations of $\mathrm{N}-\mathrm{H}$ are also calculated and are supported by the literature [18].

\subsection{0. $C-N$ vibrations}

The identification of $\mathrm{C}-\mathrm{N}$ vibrations is a difficult task, since the mixing of vibrations is possible in this region. In this study the $\mathrm{C}-\mathrm{N}$ stretching vibrations are calculated at 1131, 1106, 983, 912, 905 and $818 \mathrm{~cm}^{-1}$, respectively, and these are supported by the observed frequencies and literature [11]. The various bending and torsional vibrations assigned in this study are also supported by the literature [11].

\section{Study of intermolecular interactions using IR spectra in different phase along with different concentrations}

The FT-IR Spectra of the title molecule is recorded in the region $4000-500 \mathrm{~cm}^{-1}$. To study intermolecular interaction forces we have compared the three spectra recorded in the solid and liquid phase at two different concentrations. In general, the more condense phase gives lower frequency $\left(\nu_{\text {gas }}>\nu_{\text {liquid }}, \nu_{\text {liquid }}>\nu_{\text {solid }}\right)$. But as seen in the Table 2 , some calculated frequencies were observed in the lower region as compared to the frequencies observed in solid/liquid phase, e.g., 3602, 1336, $1241,1078 \mathrm{~cm}^{-1}$, etc. It needs to be emphasized that the calculated frequencies represent vibrational signatures of the molecules in its gas phase. Moreover, there are some limitations of the model used to calculate the vibrational frequencies, e.g., combination of electron-electron correlation and basis set deficiencies and also we have ignored intermolecular bonding because we have studied single molecule in gas phase. So on comparison of these spectra it is observed that at places where calculated frequency comes out to be lower than the experimental frequency, weak intermolecular bonding can be held responsible for it. As it can be seen from the Table 2, some lower band frequency along with higher band frequency in the case of solid CsI solution falls in the higher region than the frequency in liquid phase. In lower region, e.g., 780 and $514 \mathrm{~cm}^{-1}$ vibrational levels are close to each other, so that mixing of band occurs and it is difficult to identify the corresponding band and also in higher frequency region, e.g., 2850, 1472 and $1385 \mathrm{~cm}^{-1}$ a strikingly surprising result is observed which possibly is due to lower degree of molecular interaction, impurities present in the sample, loose packing and antibonding repulsion, etc. On comparing the vibrational spectra in two different concentrations, i.e. 5 and $10 \mathrm{mg} / \mathrm{ml}$ it can seen that nearly all frequencies lie at same level and this shows that intermolecular bonding nearly has no effect due to concentration of phase at some places in the vibrational spectra band in $10 \mathrm{mg} / \mathrm{ml}$ the frequencies lie at low region and this may be due to higher concentration and molecule have smaller space and hence cannot vibrate freely. But at some places this result reveres, e.g., 3527, 2935, 1593 and $1372 \mathrm{~cm}^{-1}$. Shift of group of frequencies can arise due to particular interaction between different molecules. Thus, the group of frequencies depends upon degree of interaction of molecules, bonding 
which lengthens and weakens the bond and hence lower frequencies. However, shift in the group of frequency position caused by resonance or intermolecular effect are in themselves highly characteristic and very useful for diagnostic purpose. We have seen that infrared spectra only appears if the vibration produces a permanent electric dipole of molecule. It is reasonable to assume that more polar the bond the more intense IR spectra arises due to the vibration of the molecule. So, it is difficult to match the intensity completely in different phases because generation of polarity depends on medium along with their concentration as well as impurity in the sample (due to difference in electronegativity). But nearly all IR intensities matches well in the same phase but different concentration.

\section{Conclusion}

The equilibrium geometries and harmonic frequencies of boldine hydrochloride were determined and analysed by QM/MM method with B3LYP/6-31G(d) and universal force field (UFF) combination using ONIOM code. The vibrational frequency calculation proved that the structure is stable (no imaginary frequencies). We found the geometry obtained by the QM/MM method to be very accurate, and we can use this rapid method in place of time consuming ab initio methods for large molecules. The difference between the observed and scaled wave number values of most of the fundamentals is very small. Any discrepancy noted between the observed and calculated frequencies may be due to the fact that the calculations have been actually done on a single molecule in the gaseous state contrary to the experimental values recorded in the presence of intermolecular interactions. The normal mode analysis of boldine hydrochloride opens up an avenue for further conformational research. With the continuing need for novel structures and the difficulty of gaining access to large tracts of bio diversity in habitats, combinatorial chemistry blended with modern quantum chemical methods can be proved to be blessing for the researchers.

\section{Acknowledgement}

The corresponding author (Neeraj Misra) is grateful to UGC (New Delhi) for providing the financial assistance.

\section{References}

[1] M. Asencio, B.K. Cassels, V. Manriquez and D. Boys, (S)-1,10-dimethoxy-2,9-dihydroxyaporphinium chloride (boldine hydrochloride), C19H22NO4+.Cl-, Acta Cryst. C52 (1996), 1581-1583.

[2] A.D. Becke, Density-functional thermochemistry. III. The role of exact exchange, J. Chem. Phys. 98 (1993), 5648-5652.

[3] S.J. Bunce, H.G. Edwards, A.F. Johnson, I.R. Lewis and P.H. Turner, Synthetic polyisoprenes studied by Fourier transform Raman spectroscopy, Spectrochim. Acta A49 (1993), 775-783.

[4] C.J. Casewit, K.S. Colwell and A.K. Rappe, Application of a universal force field to organic molecules, J. Am. Chem. Soc. 114(25) (1992), 10035-10046.

[5] S. Dapprich, I. Komaromi, K.S. Byun, K. Morokuma and M.J. Frisch, A new ONIOM implementation in Gaussian98. Part I. The calculation of energies, gradients, vibrational frequencies and electric field derivatives, J. Mol. Struct. (Theochem.) 461-462 (1999), 1-21.

[6] A. Frisch, A.B. Nielson and A.J. Holder, GAUSSVIEW User Manual, Gaussian Inc., Pittsburgh, PA, 2000.

[7] M.J. Frisch, G.W. Trucks, H.B. Schlegal, G.E. Scuseria, M.A. Robb, J.R. Cheeseman, J.A. Montgomery Jr., T. Vreven, K.N. Kudin, J.C. Burant, J.M. Millam, S.S. Iyengar, J. Tomasi, V. Barone, B. Mennucci, M. Cossi, G. Scalmani, N. Rega, G.A. Petersson, H. Nakatsuji, M. Hada, M. Ehara, K. Toyota, R. Fukuda, J. Hasegawa, M. Ishida, T. Nakajima, Y. Honda, O. Kitao, H. Nakai, M. Klene, X. Li, J.E. Knox, H.P. Hratchian, J.B. Cross, C. Adamo, J. Jaramillo, 
R. Gomperts, R.E. Stratmann, O. Yazyev, A.J. Austin, R. Cammi, C. Pomelli, J.W. Ochterski, P.Y. Ayala, K. Morokuma, G.A. Voth, P. Salvador, J.J. Dannenberg, V.G. Zakrzewski, S. Dapprich, A.D. Daniels, M.C. Strain, O. Farkas, D.K. Malich, A.D. Rabuck, K. Raghavachari, J.B. Foresman, J.V. Ortiz, Q. Cui, A.G. Baboul, S. Clifford, J. Cioslowski, B.B. Stefanov, G. Liu, A. Liashenko, P. Piskorz, I. Komaromi, R.L. Martin, D.J. Fox, T. Keith, M.A. Al-Laham, C.Y. Peng, A. Nanayakkara, M. Challacombe, P.M.W. Gill, B. Johnson, W. Chen, M.W. Wong, C. Gonzalez and J.A. Pople, Gaussian 03W, Gaussian, Inc., Wallingford, CT, 2004.

[8] R.D.J. Froese and K. Morokuma, Encyclopedia of Computational Chemistry, P. von Rague Schleyer, ed., Wiley, Chichester, Sussex, 1998, pp. 1244-1257.

[9] P. Hohenberg and W. Kohn, Inhomogeneous electron gas, Phys. Rev. B136 (1964), 864-871.

[10] D.W. Hughes, K. Genest and W. Skakim, Alkaloids of Peumus boldus. Isolation of laurotetanine and laurolitsine, J. Pharma Sci. 57 (1968), 1619-1620.

[11] V. Krishnakumar and V. Balachandran, Normal coordinate analysis of 5-fluoro, 5-chloro and 5-bromo-cytosines, Indian J. Pure Appl. Phys. 39(10) (2001), 623-627.

[12] V. Krishnakumar and R. Ramasamy, DFT studies and vibrational spectra of isoquinoline and 8-hydroxyquinoline, Spectrochim. Acta A61 (2005), 673-683.

[13] V. Krishnakumar and R. Ramasamy, Vibrational spectra and potential energy distributions for 4,5-dichloro-3hydroxypyridazine by density functional theory and normal coordinate calculations, Spectrochim. Acta A61 (2005), 25262532.

[14] V. Krishnakumar and R.J. Xavier, Normal coordinate analysis of 2-mercapto and 4,6-dihydroxy-2-mercapto pyrimidines, Indian J. Pure Appl. Phys. 41 (2003), 597-601.

[15] V. Krishnakumar, R.J. Xavier and T. Chithambarathanu, Density functional theory study of vibrational spectra, and assignment of fundamental vibrational modes of succinimide and N-bromosuccinimide, Spectrochim. Acta A62 (2005), 931-939.

[16] H. Krug and B. Borkowski, New flavonol glycosides from the leaves of Peumus boldus Molina, Pharmazie 20 (1965), 692-698.

[17] C. Lee, W. Yang and R.G. Parr, Development of the Colle-Salvetti correlation energy formula into a functional of the electron density, Phys. Rev. B37 (1988), 785-789.

[18] S. Mohan, N. Sundaraganesan and J. Mink, FT-IR and Raman studies on benzimidazole, Spectrochim. Acta A47 (1991), $1111-1115$

[19] A.K. Rappe, C.J. Casewit, K.S. Colwell, W.A. Goddard III and W.M. Skiff, UFF, a full periodic table force field for molecular mechanics and molecular dynamics simulations, J. Am. Chem. Soc. 114 (1992), 10024-10035.

[20] A. Ruegger, Neue Alkaloide aus Peumus boldus Molina, Helv. Chim. Acta 42 (1959), 754-762.

[21] H.B. Schlegel, Optimization of equilibrium geometries and transition structures, J. Comput. Chem. 3 (1982), 214-218.

[22] N. Sundaraganesn, S. Ilakiamani, H. Saleem, P.M. Wojaechiwsju and D. Michalska, FT-Raman and FT-IR spectra, vibrational assignments and density functional studies of 5-bromo-2-nitropyridine, Spectrochim. Acta A61 (2005), 2995-3001.

[23] N. Sundaraganesan, H. Saleem, S. Mohan and M. Ramalingam, FT-Raman and FTIR spectra, assignments and ab initio calculations of 2-aminobenzyl alcohol, Spectrochim. Acta A61 (2005), 377-385.

[24] N. Sundaraganesan, H. Saleem, S. Mohan, M. Ramalingam and V. Sethuraman, FT-IR, FT-Raman spectra and ab initio DFT vibrational analysis of 2-bromo-4-methyl-phenylamine, Spectrochim. Acta $\mathbf{A 6 2}$ (2005), 740-751.

[25] M. Svensson, S. Humbel, R.D.J. Froese, T. Mastubara, S. Sieber and K. Morokuma, ONIOM: A multilayered integrated $\mathrm{MO}+\mathrm{MM}$ method for geometry optimizations and single point energy predictions. A test for Diels-Alder reactions and $\mathrm{Pt}(\mathrm{P}(\mathrm{t}-\mathrm{Bu}) 3) 2+\mathrm{H} 2$ oxidative addition, J. Phys. Chem. 100 (1996), 19357-19363.

[26] A. Urzua and P. Acuna, Alkaloids from the bark of Peumus boldus, Fitoterapia 54 (1983), 175-177. 


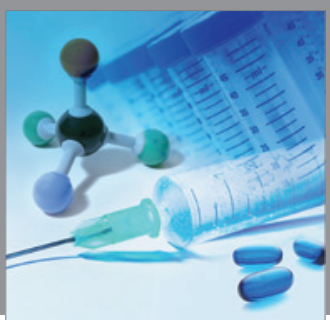

International Journal of

Medicinal Chemistry

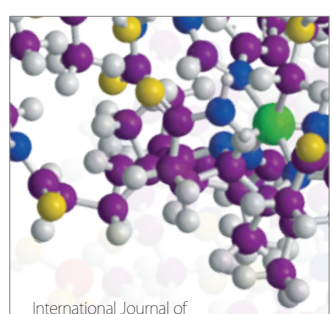

Carbohydrate Chemistry

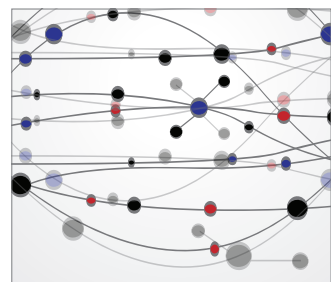

The Scientific World Journal
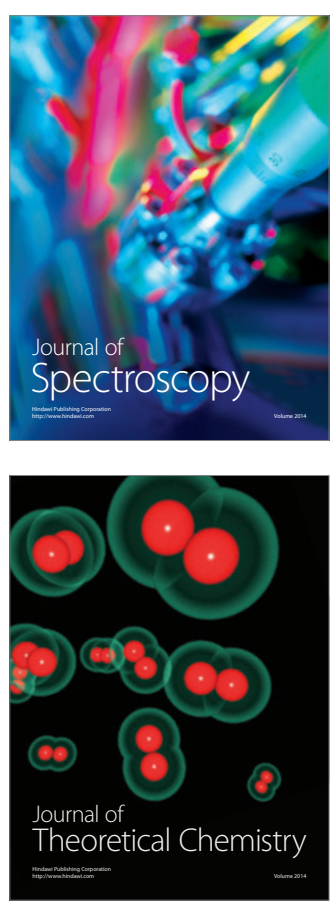
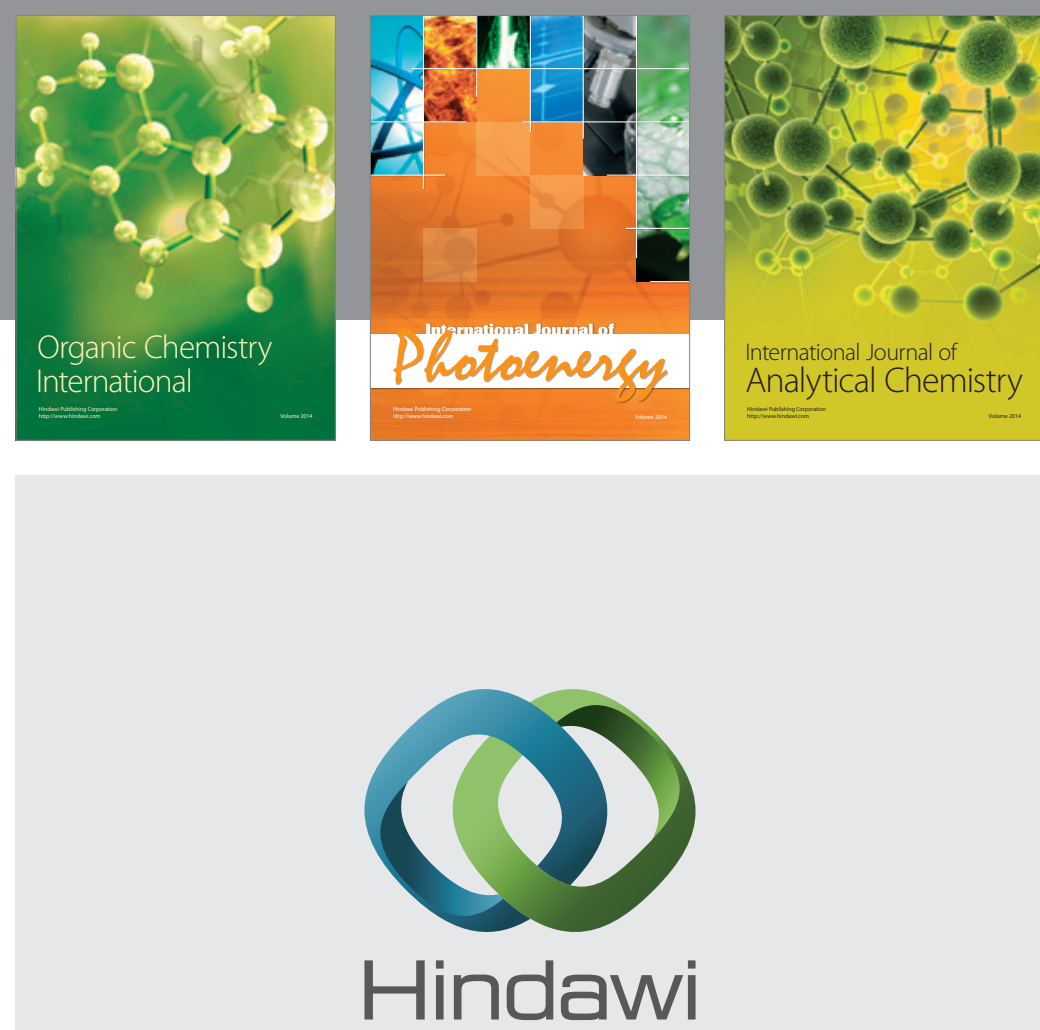

Submit your manuscripts at

http://www.hindawi.com
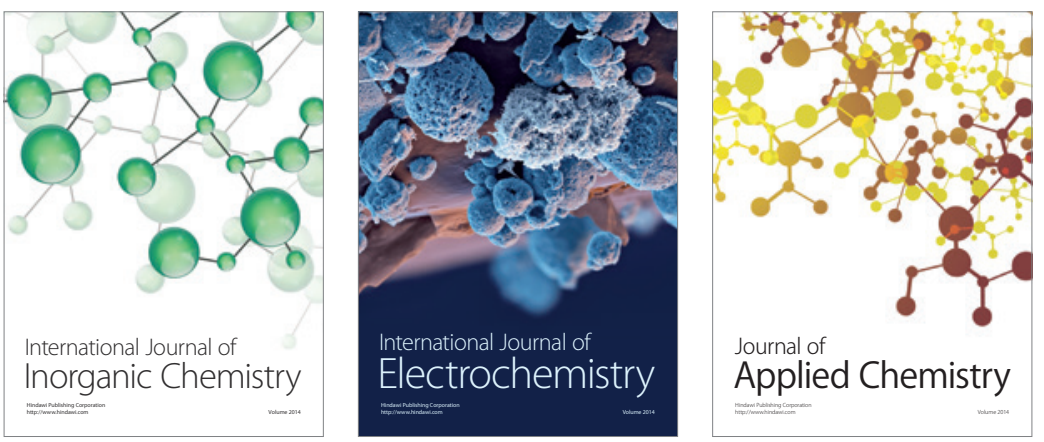

Journal of

Applied Chemistry
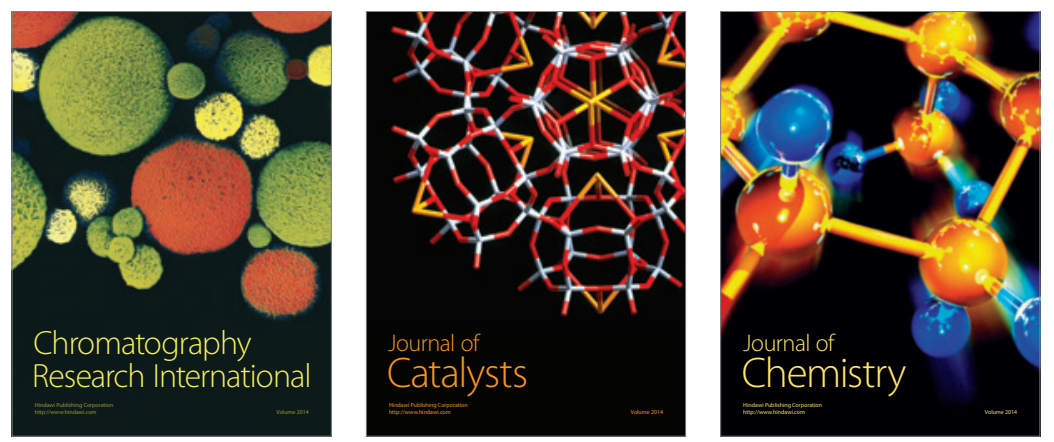
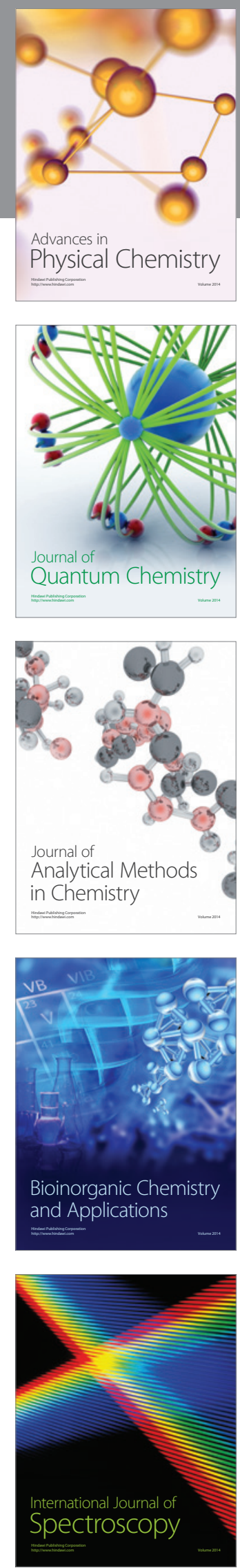